\title{
The 2020 Expert Survey on Formal Methods
}

\author{
Hubert Garavel ${ }^{1}$, Maurice H. ter Beek ${ }^{2}$ (D) and Jaco van de $\mathrm{Pol}^{3,4}(\mathbb{D}$ \\ 1 Univ. Grenoble Alpes, InRIA, CNRs, Grenoble INP, LIG, F-38000 Grenoble, France \\ 2 ISTI-CNR, Pisa, Italy \\ 3 Aarhus University, Denmark \\ 4 University of Twente, The Netherlands \\ hubert.garavel@inria.fr, maurice.terbeek@isti.cnr.it, jaco@cs.au.dk
}

\begin{abstract}
Organised to celebrate the 25th anniversary of the FMICS international conference, the present survey addresses 30 questions on the past, present, and future of formal methods in research, industry, and education. Not less than 130 high-profile experts in formal methods (among whom three Turing award winners and many recipients of other prizes and distinctions) accepted to participate in this survey. We analyse their answers and comments, and present a collection of 111 position statements provided by these experts. The survey is both an exercise in collective thinking and a family picture of key actors in formal methods.
\end{abstract}

Keywords: cybersecurity · education · formal method · modelling · safety · software engineering · software tool · specification · survey $\cdot$ technology transfer $\cdot$ verification

\section{Introduction}

FMICS, the international conference on Formal Methods for Industrial Critical Systems, is celebrating its 25th anniversary. The FMICS community recognised the revolutionary potential of verification technology quite early on. Its members devoted their energy to evolve this technology, and to apply it to the verification of complex industrial critical systems. These 25 years have brought numerous highlights, like better specification languages, more efficient verification algorithms, landmark tools, and academic recognition in the form of awards. But also many successful industrial applications, the rise of "verification engineer" as a new job title, and the advent of industrial laboratories that focus on formal verification technology.

After decades of glory, formal methods seem at a turning point. In industry, many engineers with expertise in formal methods are assigned new priorities, especially in artificial intelligence. At the same time, the formal verification landscape in higher education is scattered. At many universities, formal methods courses are shrinking, likely because they are deemed too difficult. The transmission of our knowledge to the next generation is not guaranteed. So we cannot lean back.

As part of the celebration, and in order to address this turning point, we have conducted a survey among a selection of internationally renowned scientists who have played a big role in formal methods, either within the FMICS conference series, or outside of it. We report on their collective vision on the past, present, and future 
of formal methods with respect to research, industry, and education. What did we achieve? What did we miss? Where should we go?

Related Work. Early introductions to the application of formal methods are those by Wing [22] and Rushby [19]. The 1996 survey by Clarke and Wing [8] illustrates many case studies in specification, theorem proving, and model checking. Other classical texts that reflect on the industrial application of formal methods use the metaphors of seven myths [15] or ten commandments [6].

We list a few more recent historical overviews of formal methods. A 2009 survey [23] reports about the application of formal methods in 62 industrial projects; that paper also provides an interesting overview of 20 earlier surveys on formal methods in industry from 1990 to 2009. The handbook [14] published by the FMICS community in 2012 presents applications of formal methods in various domains of industrial critical systems. An 2013 study [11] provides a synthetic account of the diverse research in formal methods, including a list of 30 carefully selected, welldocumented case studies that illustrate the progress in formal methods during the period 1982-2011. A history of 40 years of formal methods [5] includes an analysis of some obstacles to their application, while [17] focusses on their history in the UK.

Other papers examine the adoption and industrial strength of formal methods. Three recent surveys with stakeholders [4] investigate what are the most prominent formal methods styles used in the railway domain and the expectations railway practitioners have from formal tools [3]. In a follow-up experimental study [10], a panel of experts judges the suitability of nine formal methods for the specification and verification of (sub)systems in that domain. Barriers to the adoption of formal methods in aerospace are considered in a survey [9] among 31 individuals from nine organisations: the top three barriers stem from education, software tools, and the industrial environment. Multiple contributions have been made for lifting these respective barriers: [7] proposes a coherent formal methods curriculum in higher education; $[20,12]$ reflect on the development of software tools to make it more efficient and relevant, while software competitions [2] help to enhance the quality and visibility of tools; [18] provides economical evidence by demonstrating the benefits of the application of formal methods to industrial-strength problems. Finally, a recent position paper [16] discusses some obstacles and enablers for the application of formal methods, and translates them to actionable recommendations for industry, academia, and policy makers, to improve the situation.

Outline. The present report is organised as follows. Section 2 exposes the methodology used for our survey. The next five sections present and discuss the responses, which are organised in five themes: assessment of formal methods (Section 3), formal methods in research (Section 4), formal methods in industry (Section 5), formal methods in education (Section 6), and the future of formal methods (Section 7). Finally, Section 8 gives concluding remarks and Section 9 presents the 111 position statements collected during the survey. 


\section{Survey Methodology}

This section presents the main decisions concerning the organisation of the survey.

\subsection{Participants}

Initially, the plan was to centre our survey around FMICS, from its origins to our times, by asking all FMICS working group chairs, all FMICS programme committee chairs, and all FMICS invited speakers to participate in the survey. This gave a list of 94 names, much longer than that of the 1996 survey on formal methods [8], which involved 27 participants. But it became clear that our survey would benefit from an even larger panel of experts. We then started adding further names of key players in the field, based upon personal knowledge, discussions with colleagues, and taking the extensive 92-page bibliography of [11] as a source of inspiration. This resulted in a list of 230 names, which, unfortunately, was too long, since we wanted to offer each participant the possibility to write a 10-line position statement, but had only a limited number of pages in the present LNCS volume. We then devised a thorough selection procedure, based on individual scores and other criteria, in order to retain only 170 names from the list of 230 . Doing so, we tried to achieve a good coverage of academia and industry, hardware and software, global corporations and technology startups, etc., as well as a fair balance between the various styles of formal methods and a suitable geographical diversity, making sure to invite experts from most countries with a notable activity in formal methods. As the three survey organisers, we decided to exclude ourselves from the list of participants.

\subsection{Questions}

Through a long iterative process, we progressively elaborated a set of 30 questions for our survey. These questions are divided into 5 themes: assessment of formal methods (5 questions), formal methods in research (6 questions), industry (9 questions), and education (5 questions), and the future of formal methods (5 questions).

Concerning the content, most of the questions derived from our own professional experience in developing software tools, collaborating with various industries, and teaching formal methods at several universities. We also drew inspiration from other sources, among which $[11,20,12,16]$. For each question, we proposed a set of predefined, Likert-scale ${ }^{5}$ answers and, whenever possible, we added an Others alternative in case these answers would not be found relevant. We deemed many of these questions to be difficult, in the sense that we had no obvious answers for them; instead, we were curious to see all the answers given by our colleagues to figure out what was the opinion of the formal methods community on such matters. Some questions were even intentionally provocative, in order to push reflections out of the comfort zones.

\footnotetext{
${ }^{5}$ https://en.wikipedia.org/wiki/Likert_scale
} 
Concerning the form, we chose to use the open-source LimeSurvey ${ }^{6}$ software, an instance of which was already installed and freely available on an INRIA server, because this choice provided the best privacy guarantees for the experts. We thus implemented our 30 questions as an online LimeSurvey questionnaire to be filled in by the experts. For each question, we used the most appropriate LimeSurvey template, depending on whether the question had mutually exclusive answers (represented with round buttons) or multiple-choice answers (represented with square buttons). In the latter case, we often imposed a higher bound on the number of answers that experts could select, thereby forcing them to exclude approximately $33 \%$ (at least) of the proposed answers and keep only the most relevant ones. Also, whenever possible, the lists of answers were proposed in random order to eliminate option order bias (i.e. the tendency to pick the first or last answer option).

We prepared four successive beta-versions of the questionnaire and had it pretested by nine reviewers from four different countries. Their feedback helped us to improve the questionnaire through successive iterations.

\subsection{Survey}

To ease the practical management of the survey, we split the list of 170 experts into two groups of 100 and 70 people, respectively. Both groups were invited to fill in the LimeSurvey questionnaire within two successive time frames (June 3-14 and June 17-28, 2020). Each expert received one invitation and, possibly, two reminders by e-mail. In addition, intensive e-mail exchanges took place between the three survey organisers and certain experts, to provide them with more information about the survey, adapt their position statements to formatting constraints, and/or recover from technical issues with LimeSurvey (eventually, no input data was lost),

We received 130 responses after sending 170 invitations. Such a response ratio of $76 \%$ seems particularly high for an online survey. A few experts declined participation in the survey, while others remained silent. Some experts initially promised to participate in the survey, but eventually did not because they were too busy with students or peer reviews. After expiration of the deadline, in July, we also received, from a few experts, offers to participate, which we unfortunately had to decline.

In spite of the 40 missing responses, we are most happy to count, among the high-profile participants to our survey, three Turing Award winners: Hoare (1980), Emerson (2007), and Sifakis (2007); all the three recipients of an FME Fellowship Award: Jones (2015), Broy (2018), and Meseguer (2019); thirteen CAV Award winners: Alur (2008), Dill (2008), Rajamani (2011), Rushby (2012), Larsen (2013), Wang Yi (2013), Godefroid (2014), Peled (2014), Valmari (2014), Grumberg (2015), Abdulla (2017), Biere (2018), and Cimatti (2018); as well as the recipients of many other awards and distinctions that we do not list here exhaustively.

\subsection{Answers}

In total, 130 experts replied to our 30 questions. Most of them also answered a 31st additional question, which was a request to (optionally) provide a short (10-line)

\footnotetext{
${ }^{6}$ https://en.wikipedia.org/wiki/LimeSurvey
} 
position statement (cf. Section 9). The statistics recorded by LimeSurvey indicate that the mean time spent by each expert on the questionnaire was 90 minutes (and 36 seconds), while the median value was 56 minutes (and 24 seconds). Actually, the real durations are probably longer, for at least three reasons: (i) due to LimeSurvey problems, a few experts had to restart their questionnaire from scratch, and their initial attempts are not counted; (ii) many experts chose to complete their 30 answers first, and write their position statement offline to send it later by e-mail; (iii) there have been iterations with many experts to finalise their position statements. In any case, the aforementioned timing statistics represent an important collective effort from the formal methods community.

Using the LimeSurvey features, the answers of all experts were aggregated to produce, for each question, cumulative statistics, which are presented in Sections 3-7. Because it was specified that all answers to the 30 questions would remain anonymous, we considered each question in isolation and made no attempt at tracking or correlating the answers of a given expert across different questions. For the same reason, we did not try to analyse the answers using personal information about the respondents, such as country, place of work, hardware or software background, teaching activities (if any), etc.; in particular, our questionnaire did not ask for any information about the profile of participants.

\subsection{Comments}

For most questions, the questionnaire proposed a comment field in which the experts could input some text to express their opinions in more detail. Our idea was that such comments would be extremely valuable, and we intended to use them as a basis for discussing the findings of the survey, thus avoiding the pitfall of presenting statistical results only.

Such a possibility was greatly appreciated by the experts, and we received a large volume of comments (namely, 5000+ lines of 80 characters, corresponding to 111 pages of text in LNCS format) that exceeded our expectations by far. Given that all these comments could not be quoted in the present report, we had to make a selection, which raised a triage problem. A careful examination of comments led us to dispatch them into several categories:

- A critical comment expresses the dissatisfaction of the expert with the question and/or its proposed Likert-scale answers. For instance: "just a weird question".

- An explanatory comment gives the justification for the particular answer chosen by the expert. For instance: "too much irrelevant 'nice' theory".

- A restrictive comment defines the conditions in which the proposed answer is valid. For instance: "depends on the industry".

- An alternative comment provides an alternative answer (typically associated with the Other answer) and/or justifies this choice. For instance: "governments/states (through regulations)" to answer a question asking who can best drive the adoption of formal methods in industry.

- A redundant comment does not provide new information. Example: the answer "yes" accompanied by the comment "there is no doubt about this". 
- A conflicting comment introduces a contradiction with the answer it accompanies. For instance: "I chose 'probably not' but I have no opinion in fact". In such cases, we kept the answer as it was and discarded the conflicting comment. Such situations were rare and, thus, statistically negligible.

- A misplaced comment does not address the current question, but another question discussed elsewhere in the survey. Most often, "elsewhere" means "later", i.e. the respondent has anticipated on a question yet to come. In such cases, we either discarded the comment or moved it to the most appropriate question.

Such a classification was not always easy, especially for long comments (e.g. from 5 to 10 lines of text) that contained different ideas. But we did our best to process all comments and quote many of them in Sections 3-7. Some contents are ironic, or even sarcastic; mentioning them does not mean that we necessarily endorse their point of view.

The analysis of comments revealed an issue that we had not anticipated. Most questions of the survey deal with general topics such as past, present, and future of formal methods, as well as human factors, economical considerations, impact on industry and society, etc. The answers to such questions cannot be fully formal; instead, they are subjective opinions, reflected in the proposed Likert-scale options ("definitely", "probably", "probably not", "to a limited extent", etc.). Moreover, to keep the survey short and knowing that the invited experts are busy people, we tried to provide concise questions, without a lengthy set of preliminary definitions, taking as granted a number of common expressions. After submitting the questionnaire, we got some negative reactions, as the imprecision of our questions was antithetic to the culture, based on mathematical rigour, of formal methods experts. In particular, the first two questions, which we expected to be easy, made certain experts unsure and raised criticisms due to missing definitions ("what is the meaning of 'trustworthy'?"; "how do you interpret 'quality'?"; "what is the exact difference between 'partial failure' and 'partial success'?"; etc.). We believe that these questions discouraged a few experts to further consider the questionnaire.

\subsection{Terminology}

The term formal methods progressively evolved over time, starting from a narrow initial definition to a broader meaning that covers a plethora of methods and tools applied all along the design life cycle, from the elicitation of requirements and early design phases to the deployment, configuration, and run-time monitoring of actual systems. At present, formal methods encompass multiple, diverse artefacts, such as the description of the environment in which the system operates, the requirements and properties that the system should satisfy, the models of the system used during the various design steps, the (hardware or software) code embedded in the final implementation, etc. Formal methods can be used to specify these artefacts and express conformance relations between them.

Being aware of this evolution, we gave a definition of formal methods on the welcome page of the LimeSurvey questionnaire, to make sure that all respondents 
would agree on a common definition before answering the 30 questions of the survey. We adopted a modern, inclusive point of view by defining formal methods as "mathematics-based techniques for the specification, development, and (manual or automated) verification of software and hardware systems". However, when analysing the comments received (this is also manifest when reading some of the position statements in Section 9), we observed at least four different interpretations of the perimeter and scope of formal methods:

- The extensive mathematical interpretation assumes that any use of mathematics in computer science is part of formal methods. To us, this definition is too wide; for instance, the use of linear algebra in computer graphics is usually not considered to be formal methods.

- The extensive theoretical interpretation considers as "standard basic formal methods" all the concepts of formal languages, grammars, finite-state machines and automata, lexer and parser generators, etc. To us, this definition is also too wide, even if formal methods borrow many ideas from the (pre-existing) language and automata theories; for instance, the construction of a "traditional" compiler cannot be called formal methods.

- The lightweight interpretation considers as formal methods all those language features introduced for defensive programming (type checking, library interfaces, program assertions, loop invariants, pre- and post-conditions, etc.), as well as all related verifications, from simple compiler checks to advanced static analyses. Even if some concepts predate formal methods (e.g. types were already present in Algol-60), we agree that such "lightweight" techniques, which are increasingly successful in industry, are indeed part of formal methods.

- The heavyweight interpretation recognises as formal methods only those approaches that are fully mathematical and based on proofs. We consider that such a definition is too restrictive, both for design needs (in the early phases of system design, the requirements are rarely fully formal) and for practical use ("heavyweight" techniques have a clear potential, but their success stories are isolated).

Although such diverging interpretations might have affected several answers to our questionnaire, we do not see them as a serious threat to validity, given the large number of participants in the survey. But this is an important problem for the community, as it is more difficult to promote formal methods if experts do not agree on their definition. The same issue occurs at various places, e.g. in the arXiv classification $^{7}$ where formal methods must fit either under "cs.LO" (logics in computer science) or "cs.SE" (software engineering); yet, many aspects of formal methods (e.g. executable specification languages, concurrency theory, or hybrid systems) cannot easily be reduced to logics, while the numerous applications of formal methods in hardware design do not belong to software engineering. We thus call for a standalone category of formal methods, whose perimeter should be considered inclusively. As one comment wisely pointed out: "we should act as a community".

\footnotetext{
${ }^{7}$ https://arxiv.org/corr/subjectclasses
} 


\section{Assessment of Formal Methods}

\subsection{System Design}

With this first question, we wanted to query the experts about the necessity of formal methods for system design, i.e. whether or not they are dispensable or replaceable by alternative approaches.

Is it possible to design trustworthy (hardware or software) systems without using formal methods?

\begin{tabular}{|l|l|l|l|l|}
\hline Definitely: $16.2 \%$ & Probably: 21.5\% & Probably not: 33.1\% & Definitely not: $29.2 \%$ & N/A: 0.0\% \\
\hline
\end{tabular}

The answers are scattered, with no clear majority. Only the analysis of the $90 \mathrm{com}$ ments received may provide better insight.

Several comments display some criticism, since the answer depends on the definition/scope of formal methods (cf. Section 2.6), the complexity of the system, and the definition of trustworthiness. The latter is a valid point: many comments mention that a system is trustworthy only if there is an objective justification of its reliability. This interpretation introduces a substantial overlap with the next question (quality assessment). Most comments seem to agree that the question is about real systems, which are complex. We note that the answers probably not (for complex systems) and probably (for simple systems) actually express the same opinion. Five comments contradict the selected answer (maybe due to the implicit negation in without). In hindsight, a better formulation would have been: is using formal methods necessary to design well-functioning complex (hardware or software) systems?

The comments that explain that designing trustworthy systems is (definitely or probably) impossible, fall into two broad classes. The first class (14 comments) explains that formal methods are necessary to handle the inherent system complexity: "it is the size and the complexity that matter", and, consequently, that informal methods are incomplete: "it is so easy to make bugs with informal methods and extensive testing is so difficult, that adequate formal methods do help a lot in the end". The other class (14 comments) explains that trustworthy systems require some form of objective argumentation, involving unambiguous requirements. This was actually the topic of the next question. One argument was by analogy with (general) engineering. The following comment summarises these positions nicely: "The answer depends on the size, nature and complexity of software, and on the notion of 'trustworthy' you are interested in. Certainly, it is not possible to trust complex, safety critical software, built without recurring to any formalisation of its functions".

Several comments indicate that not using formal methods is possible, but infeasible or costly. "There is a very important trade off between costs, time to delivery, quality”. The comments that explain that designing trustworthy systems is (definitely or probably) possible, fall into two categories: 15 comments mention counterexamples of systems that we generally trust, but that did not use formal methods in their design, such as airplanes, while four comments even apply this to the majority of systems: "there are many examples of systems such as airplanes that are produced without the use of formal methods and in general these are still considered 'trustworthy'". Another 16 comments claim that it is possible to build trustworthy systems by 
alternative methods, such as simulation and testing, or building in redundancy, but seven comments state this is the case only for simple or non-critical systems: "properties of systems can be fully verified by exhaustive simulation if they are sufficiently small", and provided that our expectations on their reliability are sufficiently low.

\subsection{Quality Assessment}

This question also aimed to query the experts on the necessity of formal methods but, this time, for assessing the quality of complex systems.

Is it possible to assess the quality of complex (hardware or software) systems without using formal methods?

\begin{tabular}{|l|l|l|l|l|}
\hline Definitely: $15.4 \%$ & Probably: $26.9 \%$ & Probably not: 36.9\% & Definitely not: $20.0 \%$ & N/A: $0.8 \%$ \\
\hline
\end{tabular}

A majority of $56.9 \%$ deemed the use of formal methods important for quality assessment.

This question received 73 comments. Eight of them state that quality is a too broad notion that possibly includes performance, usability, process, etc., for which formal methods are not the most appropriate tool. The comments also indicate that the position of experts depends on whether systematic testing is considered to be part of formal methods or not.

There are mainly two arguments in favour of using formal methods to assess system quality. The first is that, in order to be scalable, assessment requires the use of tools, which need to rely on proper semantics: "Complex systems need scalable methods, scalable methods need a degree of automation, and such automation cannot be trusted if there is no mathematical notion of 'quality' and a model of the system supporting it". The second, more frequent, argument is that an assessment of quality requires to demonstrate the conformance of the product to an unambiguous specification: "a complex system's quality needs to be checked against well-specified requirements, and this again involves formal methods". One comment indicates that such an argument could be phrased in natural language, in principle. Another comment states: "the only way of assessing quality is by examination of the code itself, which is best conducted by specialised software based on sound theory".

Twenty-five comments mention alternative methods that can, at least, increase the confidence in digital systems: testing, simulation, statistical fault analysis, quality metrics, user interviews, and analysis of system logs. On the other hand, several comments state that alternative methods would be incomplete for complex systems, or that applying them exhaustively would be very costly ("the testing costs would be huge!"). One comment indicates that assessing the quality of the process is insufficient, although "certification institutions base their opinion mainly on criteria concerning the development process". Some comments mention certain systems considered reliable, despite not being assessed by formal methods, e.g. "Linux" and "Isabelle/HOL". Some comments distinguish quality assessment of brand new versus long-existing systems: "some military applications do surprisingly well without using formal methods. However, these are almost exclusively new variants of previously 
methods more widely, but similar arguments are also found quite often among the yes comments.

Many comments for the yes answer indicate opportunities in code analysis (e.g. avoiding memory leaks) and protocol analysis: "many cybersecurity issues involve code with memory issues, issues with access control and faulty security protocols. These would, I'd say, be typical issues that can be (and are being) addressed by formal methods". Other comments point to programming languages offering strong guarantees. Another opportunity is mentioned: "cybersecurity is particularly interesting because there are so many social factors, like social engineering, that can override verified algorithms. The challenge of how to model and verify, e.g. properties of social networks, represents an interesting frontier for formal methods".

Two comments indicate that there is much low-hanging fruit that should be harvested before applying formal methods, e.g. "programming language, architecture, development processes". There were relatively few concrete case studies mentioned, the most concrete one being the "network access restrictions [...] checked using formal methods in Azure [to] both ensure security (e.g. prevent configurations where SSH ports are opened) and avoid customer issues (detect and prevent common misconfigurations that block services)".

\subsection{Missed Opportunities}

To complete the assessment of formal methods, we wanted to know from the experts whether they believe academics have sufficiently applied formal methods.

Do you think the academic community has missed some opportunities to apply formal methods in industry, in other sciences, and/or in society at large?

\begin{tabular}{|l|l|l|l|l|}
\hline Definitely: $40.0 \%$ & Probably: $42.3 \%$ & Probably not: 10.8\% & Definitely not: $0.0 \%$ & N/A: $6.9 \%$ \\
\hline
\end{tabular}

Clearly, most experts (82.3\%) believe that some opportunities must have been missed, although, when analysing the 73 comments received, very few concrete examples are given.

Many comments put the blame either on academic practice (in particular its publication culture and its focus on theoretical results), or on industrial practice. A few comments acknowledge that the required multidisciplinarity is difficult, since academia and industry have conflicting goals. One comment describes "a healthy tension" between "on the one hand, to do as much as we can to bring formal methods to industry; but on the other, to develop intrinsically better technologies". Another comment wonders about the apparent brakes on change in industry: "why is it that we still are fighting to get accepted as a mainstream (software engineering) discipline? Why is C still the most dominant implementation language in the world?"

The probably not answer is explained in most comments by the fact that applications have been in the focus of formal methods research from the beginning: "I think there have been many serious attempts to transfer formal methods ideas to industry". Therefore, 10 comments explicitly blame industry for underestimating formal methods, e.g.: "the choice not to use formal methods can be based on silly things, such as not having the IDE one is used to". 
On the other hand, several comments state that formal methods have been oversold: "it may be the case that formal methods have been sold to industry while they were still immature". Many other reasons why we have probably or definitely missed out on opportunities were mentioned, such as the lack of standard notations, service providers, whole-system engineering approaches, support of design processes, and data-driven approaches.

Finally, only a few concrete missed opportunities are mentioned, like: "we have probably missed an opportunity to introduce formal methods in the design of medical devices"; "there are so many domains with domain-specific languages that could greatly benefit from the formal methods toolkit"; and "the formal methods community should have shown that formal methods can fit modern agile development".

\section{Formal Methods in Research}

\subsection{Overall Evaluation}

This first question polled the experts concerning the degree of success, from an academic perspective, of formal methods.

\begin{tabular}{|c|c|c|}
\hline $\begin{array}{l}\text { How would you evaluate } \\
\text { ods in academia? }\end{array}$ & the achievements of for & mal meth- \\
\hline A failure: & A partial failure: $6.9 \%$ & \\
\hline A partial success: $62.3 \%$ & A success: & N/A: $1.5 \%$ \\
\hline
\end{tabular}

The experts almost unanimously agreed that formal methods are a success or a partial success; only $7.7 \%$ stated the contrary, while a tiny minority had no opinion.

Analysing the 73 comments received, the question was largely misunderstood and criticised. One reason for this was the imprecision of the term academia (two comments mention this explicitly, e.g. "I am not sure what is meant by 'in academia'”). When drafting the question, we were interested in the perceived success of formal methods in research, but some respondents considered a different scope: 19 comments evaluate the success as partial, because of the limited success of formal methods in education ("I refer here to education, not research") and/or their lack of impact in industry. Other comments consider a partial failure and a partial success to be indistinguishable options.

The few comments from experts who consider the outcome to be a failure can be summarised as follows: "nice theory", but a lack of impact in industry "to drive formal methods into actual design, analysis, and deployment processes", and even in curricula. Note that the impact of formal methods in industry and education is addressed later in this survey using specific questions.

Further criticism, explicit or implicit, concerns how to measure success. Such ambiguity created a lot of variability in the comments, especially those considering formal methods to be a success. The most frequently used measures of success, mentioned in 12 comments, are based on objective data, such as the size of the formal methods community (considered to be an active community), the number of conferences embracing formal methods (e.g. FM, FMICS, iFM, CAV, POPL), the number 
and sociology. Formal methods for artificial intelligence hardly exist". However, this might (need to) change, since another comment notes that "the 'explainable artificial intelligence' movement $[. .$.$] cannot decently succeed without formal meth-$ ods". Six comments of experts who answered to a limited extent also mention exceptions: "human-computer interaction", "speech recognition, computer graphics, computer vision", "data science", "machine learning", and "complexity theory"; yet, another comment specifically mentions complexity theory as one of the branches for which formal methods do provide foundations.

\subsection{Main Criticisms}

This question tried to weigh to what degree the experts agree with frequently heard criticism concerning misplaced efforts of academic researchers in formal methods.

\begin{tabular}{|l|l|}
\hline $\begin{array}{l}\text { Would you agree with the criticism that most academic researchers in formal } \\
\text { methods are: }\end{array}$ \\
\hline \hline Not investing enough effort to develop software tools that are usable and robust? & $66.9 \%$ \\
\hline $\begin{array}{l}\text { Too much novelty-driven and not enough interested in the consolidation of } \\
\text { existing results to make them available to a wider audience? }\end{array}$ & $60.8 \%$ \\
\hline $\begin{array}{l}\text { Too much focussed on the most difficult and challenging problems, while neglec- } \\
\text { ting the development of broader approaches applicable to "real world" issues? }\end{array}$ & $53.8 \%$ \\
\hline Other criticism & $33.1 \%$ \\
\hline \multicolumn{2}{|c|}{ (multiple answers allowed; answers sorted by frequency) }
\end{tabular}

The three frequently heard types of criticism suggested by the proposed answers created consensus among a large number of experts, namely 87, 79, and 70 experts (in the order of frequency displayed in the table). One-third of the experts had (also) other criticism concerning academic researchers in formal methods.

This question generated the remarkable amount of 170 comments. The experts who answered other criticism had quite varying opinions, ranging from not agreeing with the proposed answers to criticising the question, typically because they believe it is difficult to generalise or because they believe neither of the suggestions belongs to the task of academic researchers. Most, however, share two general beliefs that also featured very frequently in the comments provided by those experts who did choose one of the proposed answers. Basically, the effort and interest to develop more usable and robust tools, to consolidate results and approaches and make them more widely applicable and available - clearly perceived by the experts as improving the transfer of technology to industry - is hindered by two current realities in academia: (i) a lack of academic recognition (criteria for publications and thus career promotions are based on novelty); and (ii) a lack of funding for industrial application (requiring tool advancement and maintenance). Several comments nicely summarise this belief. Some picks: "even though industry participation is sought, in essence academia creates its own bubble where criteria for success are mostly within the bubble"; "there is no business case for long term support of (academic) tools; industry needs stability and performance, academics need to innovate"; and "at the end of the day, researchers do not get much credit (nor funding) for building and 
maintaining tools and high-quality software, despite the enormous effort involved; instead, publications are more rewarded and are often what counts". This opinion recurred in 67 comments.

Finally, it is worth mentioning that two comments are positive on artefact evaluations, which "have at least accomplished that reported results are reproducible, but this is still miles away from a tool that is mature enough to be used by industry". However, one comment is convinced of the contrary: "the current practice of 'artefact evaluation' is harmful as it rewards building prototypes that are not really used by anyone, but give the illusion of building tools".

\subsection{Topic Relevance}

With this question we simply wanted to know whether the experts still consider formal methods a hot topic.

\begin{tabular}{l}
$\begin{array}{l}\text { Do you believe that formal methods are still a major topic today for academic } \\
\text { research in computer science? }\end{array}$ \\
\hline
\end{tabular}

\begin{tabular}{|l|l|l|l|l|}
\hline Definitely: $71.5 \%$ & Probably: $20.0 \%$ & Probably not: $7.7 \%$ & Definitely not: $0.0 \%$ & N/A: $0.8 \%$ \\
\hline
\end{tabular}

The vast majority of experts claimed that formal methods are indeed still a major research topic; only ten thought this is probably not the case, while one expert had no opinion. Interestingly, not a single expert thought this is definitely not the case.

This question attracted 67 comments. The seven experts who commented their choice for probably not constitute two groups of more or less equal size. One group believes that "the momentum has gone elsewhere", in one case attributing this to the fact that "industry has chosen a different direction". The other group actually seems to be convinced that formal methods are (definitely) not a major topic for research ("most computer science departments at major universities do not have anyone specialising in formal methods currently"), partly criticising the question: "still? It has been a marginal activity at most universities for a while".

Several of the 14 experts who commented their choice for probably mention that formal methods should still be a major research topic, but that it is currently "under pressure of other 'hot' topics such as artificial intelligence and machine learning". Half of the 93 experts who believe that formal methods definitely are still major research topic today added a comment, mostly explaining their choice: "though there are always certain hypes, formal methods are an important and solid basis for the development of safety-critical systems"; "in fact, formal method papers are appearing in major conferences, even outside the community. Look at the latest POPL and PLDI conferences"; and "as more and more aspects of human societies rely on some computing system, formal methods are more relevant than ever". But, there is room for improvement: "we have to learn to switch from an 'individual problem view' to a global view which exploits the power of the various methods, i.e. like going from 'post mortem verification' to 'correctness by design', which allows us to exploit the strength of many formal methods disciplines". Interestingly, one comment contradicts the opinion expressed in a previous comment: "in some public institutions the number of research teams dedicated to formal methods is relatively significant". 


\subsection{Research Priorities}

Assuming that resources for supporting research in formal methods are limited, this question asked the experts to establish a ranking between various research topics.

\begin{tabular}{|l|r|}
\hline Which should be the most urgent priorities of researchers working in formal methods? \\
\hline \hline Scalability: design more efficient verification algorithms & $70.0 \%$ \\
\hline Applicability: develop more usable software tools & $68.5 \%$ \\
\hline Acceptability: enhance integration into software engineering processes & $65.4 \%$ \\
\hline Discovery: explore new classes of problems and application domains & $44.6 \%$ \\
\hline Theory: search for the next fundamental breakthroughs & $35.4 \%$ \\
\hline Languages: design more expressive and user-friendly notations & $31.5 \%$ \\
\hline Other & $16.2 \%$ \\
\hline
\end{tabular}

(from 1 to 4 answers allowed; answers sorted by frequency)

Analysing the three most selected answers, one observes a strong wish that formal methods are applied to real problems, especially industrial ones. In this respect, the importance of scalability can be explained as the desire to overcome major obstacles to practical applications. Also, the big difference between both extremes, namely scalability and languages, might lay in the perception that the former addresses hard, objective problems deeply rooted in complexity theory, whereas the latter deals with softer, subjective issues that are largely a matter of human conventions. Such an explanation perhaps ignores the fact that languages are a key factor for industrial acceptability, and that poorly-designed languages may significantly increase the cost of formal analyses.

This question received 19 comments. Six of them refuse to define priorities, pointing out that "all the above problems are important and should be addressed" or that "science should not be priority-driven"; instead, one "should encourage researchers to follow their inspiration" and "focus on the task they are best in". One comment on scalability stresses the importance of modularity, with "compositional and reusable verification of code fragments and libraries". Two comments on $a c$ ceptability point out that the formal methods community should ensure explainability (i.e. provide "a justification for the diagnostic/result" computed by software tools) and "influence standards and regulations to make sure formal methods are required where it makes sense". Three comments on languages mention that they should be "co-developed" with verification tools and methodologies, suggesting to "design more deterministic and analysable languages (which will likely be less expressive)" and to build "good code generators" for specification languages, so as to enhance their "integration with existing programming languages". Five other comments propose alternative research priorities: validation of requirements, code synthesis, process mining, and connections to artificial intelligence, such as "artificialintelligence-driven invariant discovery".

\subsection{Software Development}

This final question on formal methods in research tries to poll expert opinions on the role and responsibility of academia with respect to the delivery of professional tools. 


\begin{tabular}{|l|c|}
\hline Which one of these two statements do you like best? \\
\hline $\begin{array}{l}\text { Public research in formal methods should only develop prototype (proof-of- } \\
\text { concept) tools, while leaving the development of professional tools to industry }\end{array}$ & $36.2 \%$ \\
\hline $\begin{array}{l}\text { Formal methods are too involved and their market is too small for most compa- } \\
\text { nies, so academia should invest effort to develop and consolidate usable tools }\end{array}$ & $38.5 \%$ \\
\hline Other answer & $25.4 \%$ \\
\hline
\end{tabular}

This question apparently divided the experts: while one-fourth did not like either of the two statements best, we note an almost perfect distribution of the remaining experts among the two statements. The outcome thus provides little guidance as to where the effort concerning professional tool development should come from.

This question received 80 comments, most of which are insightful. The 34 comments provided with other answer actually show a remarkable variety of opinions. Ten comments believe (to a certain extent) in both: "the tools we develop should be usable (and extensible) by researchers in our own community, and should therefore go beyond the proof-of-concept stage. However, we should not spend time on polishing the things that matter for acceptance in industry, such as user interfaces, have round-the-clock available help desks, liability, etc.". Ten comments (strongly) believe in neither of the two statements. Five comments believe in a "combination of the two statements": "neither fully, both partially". Another ten comments believe something similar, namely that developing formal methods tools should be a collaborative effort by academia and industry, but four of them note that academia should be leading the development, and five of them that academic prototypes "should go beyond the current state of proof-of-concept tools". A couple of comments, finally, mention that "effort should be devoted to open-source community efforts".

The 16 comments provided by those experts who best like the first statement are very much in line, mainly indicating two (related) reasons. First, eight comments claim that tool development is "not the role of academia" and "most academic institutions are not equipped to maintain professional level tools, even if they manage to develop a first version". Second, four comments claim there is a lack of "funding to develop industrial-strength tools".

The 30 comments provided by the experts preferring the second statement are less in line, but there are two recurring reasons in support of this statement. First, eight comments state that "good research groups tend to work on one tool, for decades, which brings about solid tools"; CADP, UPPAAL, and Z3 are explicitly mentioned as examples. Second, six comments state that "this is the only way to provide technological transfer to industry, [as] in most cases efficient implementation requires to know a bit about the underlying theory".

\section{Formal Methods in Industry}

\subsection{Impact Evaluation}

This first question asked from the experts to evaluate the degree of success of formal methods in industry. 


\begin{tabular}{|l|l|}
\hline $\begin{array}{l}\text { How would you qualify the impact of formal methods on } \\
\text { industrial software development practices? }\end{array}$ \\
\begin{tabular}{|l|l|l|}
\hline A failure: $2.3 \%$ & A partial failure: $29.2 \%$ & \\
\hline A partial success: $63.8 \%$ & A success: $3.1 \%$ & N/A: $1.5 \%$ \\
\hline
\end{tabular}
\end{tabular}

According to most answers, the impact is neither a complete success nor a complete failure, but in between, and clearly more of a success than a failure.

This is confirmed by the 79 comments, which are distributed as follows among the proposed answers: 0 for failure, 24 for partial failure, 51 for partial success, 3 for success, and 1 for no opinion. Eighteen comments mention "a few great achievements" of formal methods in CAD and "EDA tools for hardware design and embedded software", "in a few enlightened industries (aerospace, railway, nuclear)", "in some fields like avionics, distributed algorithms, and now security", and in "many of the most successful companies", which "develop and adopt formal methods for [their] own use" - with mentions of Airbus, AMD, ARM, ASML, AWS, Facebook, Google, Huawei, IBM, Intel, Microsoft, Philips Healthcare, and Siemens, "just to name a few". Building a global picture is difficult however, since "some of the work is being done by secretive companies who do not publish/highlight their successes": formal methods are often used "behind the scenes" and "unfortunately, once a formal-methods tool becomes successful, it [is] usually renamed to something else".

Twelve other comments list "ideas of formal methods [that] found their way into modelling and programming languages", e.g. "typed languages (in spite of the current Python frenzy)", "type checking" and "type inference", "interfaces for libraries", "assertions in programs", "pre- and post-conditions of functions", but also "techniques that improve the code production", e.g. "model checking", "automatic test case generation", "lightweight verification tools (runtime verification, 'linters', etc.)", "static analysis and other embedded analyses, [which] are accepted and included in industrial toolchains", and "now routinely used for systems software and opensource software", sometimes "without people even realising it".

On the critical side, forty comments express almost the same idea: "formal methods are [...] used only to a limited extent, and not where it would be required"; "in a few industries it has been a success but in the majority of industries not"; "there are some successes to celebrate, but they are at the moment too few to have impact". One comment underpins this general opinion: "there are some ways to measure this impact: the offers to hire professionals with formal-methods background, the investment of software licenses for formal-methods-based tools, the contracts with research institutions or companies to solve specific projects, etc. I do not see a big impact with these parameters".

Some comments justify the fact that many companies are not using formal methods either for financial reasons ("too little of a cost reduction in return for too great an investment of time and skill"; "company cultures [are] particularly hostile to things that give no immediate product, but merely add quality to a product"), or due to human factors ("the inertia of industrial software development practices is enormous"; "the somewhat heavy emphasis on having a rich math background [...] is not going to be mainstream"), or by historical after-effects ("formal methods may have gotten a 'bad reputation' [since they] in some cases have become associated with '1980s 
in productivity and quality". "They can be a useful bug finding tool. Ideally, they will be integrated into IDEs and compilers and operate in the background".

Fifteen comments note that formal methods are only ready to be applied by sufficiently skilled and willing people: "there is probably still a lack of trained engineers and of will" and "it also requires scientific skill and attitude". There are conflicting comments around "being modest". On the one hand: "it may still be too early for a wide-spread roll-out of formal methods in industry. We run the risk of overpromising", but, on the other hand: "how many whip lashes should you self-apply before you have the permission to venture out in the world?"

\subsection{Return on Investment}

This question asked the experts to make an informal cost-benefit analysis over time.

\begin{tabular}{|lr|l|r|}
\hline In your opinion, are formal methods profitable enough to outweigh their costs? \\
\hline No return on investment: & $2.3 \%$ & Profitable in the long term only: $12.3 \%$ & \\
& \\
\hline Immediately profitable: & $15.4 \%$ & Profitable in medium and long terms: $58.5 \%$ & N/A: $11.5 \%$ \\
\hline
\end{tabular}

A small majority judged that the application of formal methods is profitable in medium and long terms. Another 15\% (resp. 12\%) indicated that they pay off immediately (resp. in the long term). A few experts answered that formal methods do not pay off, while a relatively large group has no opinion.

This question received 73 comments. In the no opinion category, two comments criticise the question as ill-posed: "your scale is very unhelpful", or even: "your question is part of the problem". The other ten, however, indicated that the answer depends too much on the specific circumstances.

The comments justifying immediate pay-off are very diverse. Some see the payoff in the added value, either "to explore and analyse design-time problems", or as an alternative to "more ad-hoc methods such as testing", or in "added security and safety". Others justify the pay-off by the huge costs of errors in critical software. Three comments condition an immediate pay-off on the proper alignment with software development processes, for instance: "the key is to align the formal methods [...] with incremental software development".

Ten comments explicitly mention that initial investment costs prevent an immediate return on investment: "as for any technology move, one needs to adapt methods and tools, to train and educate, to practice". One comment concludes that "the initial cost is really high, and a critical research focus should be on how we can provide lightweight formal methods that are more proportional in their effort/value ratio", while another expects that "if smoothly integrated into the development process, the extra cost will be amortised by the savings gained from better quality". Indeed, several comments point out that "the real savings [come] later with improved product quality and reduction of errors".

But "a clear problem is that the benefits cannot be quantified clearly", especially when "companies get away with the consequences of their bad development", "as long as states/governments do no enforce strict regulations with proper penalisation". Four further comments explain that the real cost savings appear only later, 
with less and cheaper maintenance due to fewer failures. Another fifteen comments note that the return on investment "is really depending on the context, and the right choice of technique and problem".

Another argument justifies long-term-only benefit after considerable investment: "we should think of formal methods as a 'disruptive technology'. Such technologies have the potential to change the way things are done and generate a process of 'creative destruction' in Schumpeter's sense; but this of course generates resistance and requires investment, more than of money, investment on people".

\subsection{Most Effective Framework}

This question polled the experts to know in which companies formal methods can be most efficiently deployed.

\begin{tabular}{|l|r|}
\hline \multicolumn{2}{|l|}{ Which kind of company is best suited for using formal methods? } \\
\hline $\begin{array}{l}\text { Large companies, because they have the budget and time frame needed } \\
\text { to experiment with formal methods }\end{array}$ & $23.8 \%$ \\
\hline $\begin{array}{l}\text { Small companies, because they are agile enough to prototype with non- } \\
\text { standard languages and software tools }\end{array}$ & $6.9 \%$ \\
\hline Any kind of company, whatever its size & $63.8 \%$ \\
\hline N/A & $5.4 \%$ \\
\hline
\end{tabular}

The majority of the experts (around 70\%) did not select a clear advantage for either large or small companies, when it comes to the application of formal methods. The number of experts that expect a fruitful application from large companies was three times larger than the number of experts who expect this from small companies.

Looking at the 61 comments received, 20 of them indicate that the presence of skilled and enthusiastic people is more important than company size. Another 13 comments express that the application domain is more important than company size. These reasons can explain why many experts did not choose any of the extremes: "the size of the company does not matter. What matters is their implicit motivation (to be the best in the business), the ability of a local champion (to carry the torch, overcome internal hurdles, motivate other people), and an obvious business opportunity where the application of a formal technique is of paramount benefit".

The comments provide further insights in the perceived difference between small and large companies. On the one hand, eight comments indicate that large companies are more suited, as they can devote time and budget to formal methods: "large companies are typically willing to invest in pilot projects to study the feasibility of using formal methods. They have the financial means to do so". On the other hand, eight comments indicate that small companies are more agile to adopt formal methods quickly: "small companies can decide faster and are more dependent on quality"; "the actual killer case would be a startup company formed of people who are already highly trained in formal methods and have a killer app for which formal methods gives them overwhelming advantage". Three other comments discuss examples of formal methods deployed in small or big companies. 


\subsection{Limiting Factors}

This question asked the experts to rank a large number of potential barriers and obstacles that may prevent formal methods from being accepted in industry.

\begin{tabular}{|l|r|}
\hline What are the limiting factors for a wider adoption of formal methods by industry? \\
\hline \hline Engineers lack proper training in formal methods & $71.5 \%$ \\
\hline Academic tools have limitations and are not professionally maintained & $66.9 \%$ \\
\hline Formal methods are not properly integrated in the industrial design life cycle & $66.9 \%$ \\
\hline Formal methods have a steep learning curve & $63.8 \%$ \\
\hline Developers are reluctant to change their way of working & $62.3 \%$ \\
\hline Managers are not aware of formal methods & $57.7 \%$ \\
\hline Many companies do not pay enough attention to software quality and security & $56.2 \%$ \\
\hline Formal methods are not properly integrated in standard certification processes & $46.9 \%$ \\
\hline Formal methods focus on relevant problems, but only on a small part of all problems & $36.9 \%$ \\
\hline Benefits of formal methods are not marketed enough & $36.9 \%$ \\
\hline There are too many formal methods, with a lack of independent comparison & $28.5 \%$ \\
\hline Formal methods are too costly, with no perceived immediate added value & $26.9 \%$ \\
\hline Formal methods are too slow to meet current time-to-market constraints & $17.7 \%$ \\
\hline Professional tools are too expensive because of the small market for them & $14.6 \%$ \\
\hline Other approaches to software quality outperform formal methods & $13.1 \%$ \\
\hline Industrial software development practices change too often and too quickly & $8.5 \%$ \\
\hline Formal methods focus on the wrong problems & $7.7 \%$ \\
\hline Other & $13.8 \%$ \\
\hline
\end{tabular}

(from 1 to 12 answers allowed; answers sorted by frequency)

Interestingly, obstacles arising from human factors predominate, as the 1st, 4th, 5th, and 6th most selected answers reflect educational problems, namely a lack of knowledge from managers and developers, and their difficulties to learn and deploy formal methods. Technical factors appear in the 2nd and 3rd answers, whereas financial factors underlie the 7 th answer.

This question attracted 17 comments, most of which are attached to the other answer but actually correspond to answers already present in the above list (namely, the 1st, 2nd, 4th, 5th, and 8th most selected answers). For instance, five comments echo the 1st answer (engineers lack proper training in formal methods), one of them regrets that "education in formal methods frightens off students and puts them off for life rather than showing potential benefits". Two comments, somewhat related to the 5th answer (i.e. developers are reluctant to change their way of working), raise concerns about misguided applications of formal methods: "formal methods people are too stubborn; they advocate that everything should be formal", but "trying to apply formal methods everywhere is a non-sense", as "formal methods have to be sold [only] to people with problems". Two other comments reinforce the 8th answer (i.e. formal methods are not properly integrated in standard certification processes), regretting that "professional bodies do not encourage best practices, like they do in other disciplines" and that "regulation often focuses on process quality, not product 
quality". Another comment draws a critical eye on those limiting factors: "it is like benefits that show in the long-term, they are trumped by short-term obligations".

\subsection{Research-Industry Gap}

This question tried to evaluate the distance, and its growth trend, between the problems actually faced by industry and the solutions brought by academic researchers.

\begin{tabular}{|l|r|}
\hline Which one of these assertions do you consider to be the most appropriate? \\
\hline \hline There is no gap between academic research in formal methods and industry & $2.3 \%$ \\
\hline There is currently a gap, but it is narrowing & $68.5 \%$ \\
\hline There is currently a gap and it is growing & $20.0 \%$ \\
\hline N/A & $9.2 \%$ \\
\hline
\end{tabular}

Most experts agreed upon the existence of a gap between academic research and industry, and they are also positive that this gap is getting smaller.

This question received 54 comments distributed as follows among the proposed answers: 3 for no gap, 33 for narrowing gap, 13 for growing gap, and 5 for no opinion. One comment indicates that "a general response" to such "a very open question" is impossible. Three comments point out that "in hardware companies there is virtually no gap", as these "companies are long-time users of formal methods"; so, most of the discussion focuses on software and systems development. A large majority of comments consider that the gap is narrowing, since "technology transfer is visibly increasing", but five comments notice that "progress is slow" or "very slow", and "there is a huge work to be still done". Other comments make a clear distinction between "a few elite companies" (Amazon, Facebook, Google, Microsoft, and Thales Railways are cited in four comments as "examples of the gap narrowing"), certain application domains ("in hardware design, communication protocols, critical applications like avionics, and formal system testing, [the] gap seems to be narrowing"), and the rest, for which "the situation is heterogeneous", as "more industries get interested" in formal methods, "but few do more than experiments". Formal methods are also successful in domains such as "cryptocurrencies, [where] any bug can cause an enormous financial loss". The market size, in itself, does not seem to be a criterion: "the gap is narrowing in safety-critical robotics [but] growing in Android mobile-phone apps; both are multi-billion dollar industries".

The explanations given for a growing gap are threefold: complexity of industrial projects and agility of industrial processes ("industry is moving forward very fast, and academia has a hard time to catch up"), fragmentation of formal methods ("too many competing approaches with too little distinguishing impacts in practice", as well as increasingly complex "extensions of [...] computational models that are only relevant to increasingly smaller audiences"), and lack of properly trained personnel ("the education of software professionals seems to contain less and less hard topics such as logic"). This latter point is deemed crucial, as "the dismal lack of mathematical abilities of the iPhone generation and the dismantling of theoretical courses [...] even in top universities" prevents the gap from being filled ("if you always need the 
academic doctor working in formal methods for a real industrial project, then something is wrong").

Six comments confirm that "there is a gap" but one "cannot tell whether it is narrowing or growing", because "while formal methods are becoming more mature and capable to handle larger problems, problems are also becoming more complicated". Finally, a comment suggests that the gap is perhaps different from what one would expect, as "Google, Facebook, and Amazon have stronger formal-methods research than most academic groups", whereas another comment recommends that academic research "should make progress regardless of industry, as long as there are realistic applications".

\subsection{Design Life Cycle}

It has often been stated that formal methods are best applied all the way, step by step, from the initial requirements to the final executable code. However, many publications report successful uses of particular formal methods in particular phases of the design life cycle. This question tries to explore and quantify the discrepancy between the ideal expectations and the practical achievements.

\begin{tabular}{|c|c|}
\hline $\begin{array}{l}\text { In which phases of the design life cycle are formo } \\
\text { likely to be the most useful? }\end{array}$ & ethods \\
\hline Generating test cases, especially for corner cases & $77.7 \%$ \\
\hline Capturing and formalising requirements & $75.4 \%$ \\
\hline Checking whether models are correct & $69.2 \%$ \\
\hline Building models of the system & $64.6 \%$ \\
\hline Validating the requirements & $53.8 \%$ \\
\hline Generating code from models & $53.1 \%$ \\
\hline Certifying correctness of the final code & $45.4 \%$ \\
\hline Monitoring deployed software at run time & $43.1 \%$ \\
\hline Maintaining consistency between models & $42.3 \%$ \\
\hline Detecting mistakes in handwritten code & $39.2 \%$ \\
\hline Evaluating the test results & $20.0 \%$ \\
\hline Other & $10.8 \%$ \\
\hline
\end{tabular}

The presence of test-case generation at the top of the list is significant, as it contradicts the ideal vision of a fully formal design flow, where refinement is used at each step to ensure that the final code satisfies the initial requirements. Indeed, in such a design flow, tests would be no longer necessary or, at least, their importance would decrease. Instead, the stated relevance of formal methods for test-case generation indicates that formal methods fit well with conventional design flows, in which testing efforts often represent more than a half of the total development costs. Cutting down such efforts (e.g. by generating tests automatically and/or by generating tests of a better quality) is thus a promising target for formal methods. The next answers in the list show that different methods can be beneficially used during the various 
phases of the design life cycle. All in one, the answers suggest that formal methods can be evolutionary, rather than revolutionary.

This question received 13 comments, all associated with the other answer. A first group of comments stresses that formal methods should be used in all phases of the life cycle to maintain some consistency from requirements to code. A second group of comments suggests other specific uses of formal methods: "certified compilation", "deployment configuration", analysis of "legacy systems", assurance that "certain classes of bugs" are "absent [from] the final code", and development and verification of "concurrent and distributed systems" and "systems of systems".

\subsection{Dissemination Players}

The next question tried to determine who, in the stakeholder network that exists between academia and industry, can contribute most to the industrial deployment of formal methods.

\begin{tabular}{|c|c|}
\hline \multicolumn{2}{|c|}{$\begin{array}{l}\text { Who could best drive a more widespread application } \\
\text { of formal methods in industry? }\end{array}$} \\
\hline Universities and engineering schools & $63.8 \%$ \\
\hline Research and technology institutes & $63.8 \%$ \\
\hline Large industrial companies & $50.0 \%$ \\
\hline Tool-vendor companies & $46.2 \%$ \\
\hline Dedicated service companies & $30.0 \%$ \\
\hline Others & $14.6 \%$ \\
\hline
\end{tabular}

The answers make it clear that all stakeholders have a role to play, perhaps at a different level and with a different impact factor. Somewhat paradoxically, the respondents show greater confidence in public (or non-for-profit) institutions than in private companies, although the goal is to trigger methodological changes in entities belonging to the private sector - a trend that is in line with former answers, such as those of Section 4.6.

This question attracted 131 comments distributed as follows among the proposed answers: 29 for universities and engineering schools, 27 for research and technology institutes, 24 for large companies, 20 for tool-vendor companies, 13 for dedicated service companies, and 18 for others.

Concerning universities and engineering schools, their most important mission is, according to 17 comments, to "create the necessary critical mass of talent-pool" by "delivering more graduates who know and like formal methods". Yet, five comments point out that "these institutions should be doing it better" and "definitely enhance their commitment in formal methods". Four comments list research-oriented missions, such as "illustrating novel ideas", "demonstrating the state-of-the-art via prototypes", and "develop[ing] and maintain[ing] formal methods tools".

Concerning research and technology institutes, four comments cite the examples of Fraunhofer (Germany), GTS (Denmark), INRIA (France), IRTs (France), MPI (Germany), MSR (worldwide), and SRI (California). Six comments point out that such 
institutes "can play an important role in industrial take-up of formal methods" since "they are at the interface between researchers and industry" and, thus, "have more chance to be closer to the problem domain". Ten comments expect them to "play a crucial role" in "devising user-friendly formal methods, designing efficient analysis methods, and developing robust tools", and in "taking up larger challenges" to "demonstrate the value of formal methods on actual systems". Two comments claim that such institutes "are better at long-term investment than individual universities", but "they have to realise the missions set to them by their paymasters".

Concerning large companies, seven comments mention Airbus, Amazon Web Services, Facebook, Google, Intel, Microsoft, and Thales, as well as "organisations in general that build critical software systems". Eleven comments consider such companies and organisations as ideal hosts for formal methods: "they have the money, they have staff to spare, they have problems at scale, and they have the visibility that when they speak up, others listen"; this latter point references their capacity to "champion formal methods" and "boost the[ir] widespread application" by "commit[ting] their suppliers" and "provid[ing] a market for tool vendors". However, two comments warn that large companies "do have the resources, but are often slow to react", so that "we keep seeing companies on the brink of bankruptcy due to catastrophic errors that formal verification could catch".

Concerning tool-vendor companies, two comments stress the importance of software: "without tools, no application of formal methods". Nine comments state the missions expected from such companies: "transfer academic ideas and prototypes to industrially applicable software tools", "sell and maintain [these] tools" and "make [them] appealing" by "working on usability issues", "provide tutorials and courses", and "offer consultancy" services. Two comments consider tool vendors as "companies that are quite successful", while three other comments predict that such companies "have the heaviest resources and motivation to promote formal methods", and that "tool vendors that open up the market can make a difference" and "will ultimately decide the acceptance of formal methods".

Concerning dedicated service companies, five comments discuss the business model and genesis of such companies, which can be either "started up by academics" or spun off from larger companies that "prefer outsourcing this activity, at least temporarily". Seven comments define such companies as arrays of "consultants", who "concentrate a critical mass of expertise" and "specialised knowledge" to "help choosing the most appropriate approach" and deliver "formal methods as a service".

Concerning others, five comments do not give a precise answer, while other comments suggest further stakeholders who could contribute to the industrial adoption of formal methods: governments/states (through regulations), certification authorities (through quality standards), funding agencies, alliances for open source and open APIs, non-for-profit associations, and communities of software developers.

\subsection{Academic Policies}

The last question of this group reviewed the concrete actions academia can do to improve the transfer of formal methods results to industry. 


\begin{tabular}{|l|l|}
\hline $\begin{array}{l}\text { Which academic policies can contribute most to the adoption of formal } \\
\text { methods in industry? }\end{array}$ \\
\hline More collaborative projects between research and industry & $78.5 \%$ \\
\hline Increased support for academic researchers developing tools & $68.5 \%$ \\
\hline Construction of benchmarks and datasets for formal methods & $53.1 \%$ \\
\hline Construction of learning resources for formal methods & $48.5 \%$ \\
\hline Dedicated engineers to increase the quality and TRL ${ }^{8}$ of academic tools & $45.4 \%$ \\
\hline Databases of case studies showing the applicability of formal methods & $44.6 \%$ \\
\hline Collaborative software platforms integrating tools from different institutions & $35.4 \%$ \\
\hline Economic studies to estimate the return on investment of formal methods & $34.6 \%$ \\
\hline Increased resources and scientific credits to software competitions & $26.9 \%$ \\
\hline Others & $10.8 \%$ \\
\hline
\end{tabular}

The analysis of the most selected answers shows three main lines of action for academia: (i) collaborative projects with industry, the number of which should be increased; (ii) software tools, for which academia should receive greater financial and human support - notice that such a confirmation of the manifest role of academia in tool development corroborates the prior results of Section 4.6; and (iii) scientific data, by producing benchmarks, datasets, case studies, and learning resources.

This question received 14 comments, most of which associated with the others answer. Four comments recommend to "invest in long-term collaboration with industry" (as opposed to the usual short-term projects supported by funding agencies), with "academic reward structure changes", "increased support and scientific credit for researchers involved in collaborative projects with industry", and "programs for $\mathrm{PhD}$ theses to be done in collaboration between academia and industry". However, another comment warns that "formal methods will [only] succeed in industry when a CEO decides it is a priority", a possible reminiscence of Bill Gates's famous memo on security [13]. Two other comments evoke the "inclusion of formal methods in regulatory regimes", with "standards and regulations that demand the kind of guarantees that only formal methods can provide". Finally, four other comments mention educational issues (specifically addressed in Section 6 below), with the suggestions of "updating curricula in ICT professionals at bachelor level" and "teaching students on a large scale", with a "compulsory formal methods module" and "better courses that speak to students' needs rather than professors' passions".

\section{Formal Methods in Education}

\subsection{Course Level}

Our first question concerning education was to ask the experts about the most suitable place for formal methods in an ideal teaching curriculum.

\footnotetext{
${ }^{8}$ Technology Readiness Level (http://en.wikipedia.org/wiki/Technology_readiness_level).
} 


\begin{tabular}{|l|r|}
\hline When and where should formal methods be taught? \\
\hline \hline In master courses at the university & $80.0 \%$ \\
\hline In bachelor courses at the university & $79.2 \%$ \\
\hline In professional (software) engineering schools & $70.8 \%$ \\
\hline In continuing education for professionals & $70.0 \%$ \\
\hline During doctorate studies & $31.5 \%$ \\
\hline Others & $3.8 \%$ \\
\hline
\end{tabular} (from 1 to 4 answers allowed; answers sorted by frequency)

The main lesson is that formal methods should be taught early, in bachelor and master courses. Waiting until doctorate studies would be a mistake, as the $\mathrm{PhD}$ students would not have enough time to acquire a proficiency level in formal methods sufficient to survive on the international research scene.

There were only five comments on this question, all associated with the others answer. Most of them indicate that formal methods should be taught in all the proposed answers. Another comment points out that non-specialists should be taught "mathematical thinking and the capacity of abstraction, not formal methods per se".

\subsection{Importance Level}

This question asked the experts about the current situation of formal methods in computer science teaching. In order to avoid no opinion answers from respondents lacking a global overview of universities, we added a restriction to familiar schools.

\begin{tabular}{|l|r|}
\hline $\begin{array}{l}\text { What is your opinion on the level of importance currently attributed to } \\
\text { teaching of formal methods at universities? (If you feel that the question } \\
\text { is too general, restrict your answer to the universities you know best.) }\end{array}$ \\
\hline Not enough attention & $50.0 \%$ \\
\hline Sufficient attention, but scattered all over & $31.5 \%$ \\
\hline Right level of attention & $6.9 \%$ \\
\hline Too much attention relative to other skills & $1.5 \%$ \\
\hline No opinion & $10.0 \%$ \\
\hline
\end{tabular}

Exactly half of the experts indicated that formal methods do not receive enough attention in university curricula, while roughly one-third expressed it does, but in a scattered way. Only nine experts responded that universities attribute the right level of attention to teaching formal methods, while two experts answered it receives too much attention. Thirteen experts had no opinion.

This question received 47 comments. The four comments expressing no opinion mention that the answer varies too much "from country to country and institution to institution". Four of the six experts commenting on the right level of attention base their opinion only on their personal situation. These two types of comments are also common for the not enough attention and sufficient attention, but scattered answers.

Another recurring comment is that education in formal methods is often isolated. Five comments indicate that applications of formal methods should occur in other courses, like databases, algorithms, concurrency, distributed systems, operating systems, security, compilers, and programming languages. A few comments also 
mention that the role of formal methods in the software development process and in actual engineering practice should be taught. Another comment explicitly mentions that formal methods should be given "the same relevance as programming".

Finally, the comments also point to some causes of the under-representation of formal methods in curricula: unawareness among staff and management, emergence of new hypes (e.g. heuristic and agile approaches), computer science curricula getting more and more crowded with other topics, and students of an increasingly variable entrance and abstraction level.

\subsection{Course Format}

This question investigated the target audience and the most appropriate contents for formal methods courses.

\begin{tabular}{|l|r|}
\hline Which of the following course formats is preferable? \\
\hline $\begin{array}{l}\text { Intensive courses on formal methods, targeted to a small number of good students, } \\
\text { so as to ensure that the research in formal methods remains strong }\end{array}$ & $6.9 \%$ \\
\hline $\begin{array}{l}\text { Non-specialist courses giving a flavour of formal methods combined with other top- } \\
\text { ics (software engineering, distributed systems, embedded systems, etc.) }\end{array}$ & $5.4 \%$ \\
\hline $\begin{array}{l}\text { Both: specialist courses taught to a limited number of students, and gentle introduc- } \\
\text { tion to formal methods for a larger number of students }\end{array}$ & $83.8 \%$ \\
\hline Other answer & $3.8 \%$ \\
\hline
\end{tabular}

The answers show a clear consensus of the experts on the 3rd answer (both), with an overwhelming majority.

This question received 32 comments, distributed as follows among the proposed answers: 4 comments for intensive courses, 2 comments for non-specialist courses, 21 comments for both, and 5 comments for other answer. Three comments decline to answer the question, arguing that "it very much depends on the level and of the kind of students" and that "each instructor has to figure this out". Five comments state that "every bachelor in computer science/informatics should know about formal methods" and "be trained in applying [them]". Four comments stress that "intensive courses [taking] the matter seriously are the only way to truly educate people", whereas "overview courses (gentle introductions)" giving "just a flavour [are] more likely to lead to disappointment than to something good". Another comment warns, however, that "students (and practitioners) will avoid intensive courses as long as [...] they are too complex". Eleven comments support the two-level approach proposed by the 3rd answer (both), putting forward the need to educate a few specialists, who will design new methods and tools, as well as a majority of software engineers and future managers, who will adopt these methods and tools in their professional practice. Finally, one comment recommends to "spread the word" about the excitement of formal methods: "it is not a religion, but treating it as such may help".

\subsection{Course Organisation}

The next question asked about the best manner to set up formal methods courses. 


\begin{tabular}{|l|c|}
\hline How should formal-method courses be organised? \\
\hline \hline $\begin{array}{l}\text { Top-down: primarily focused on theory, starting from theoretical results and } \\
\text { possibly illustrating them with applications }\end{array}$ & $6.9 \%$ \\
\hline $\begin{array}{l}\text { Bottom-up: starting from concrete problems, and later introducing theory as a } \\
\text { means to solve these problems }\end{array}$ & $40.8 \%$ \\
\hline $\begin{array}{l}\text { Alternative way, possibly with a combination of top-down and bottom-up (you } \\
\text { can explain your vision in the comment field) }\end{array}$ & $44.6 \%$ \\
\hline Other answer & $7.7 \%$ \\
\hline
\end{tabular}

Only nine experts answered a top-down setup would be best, but the vast majority was divided between a bottom-up setup and an alternative way.

Fortunately, the analysis of the 58 comments provides more detailed information. Ten experts chose other answer, but six of them actually suggested an alternative way in their comments, often even a mixture of top-down and bottom-up. Besides, two experts who opted for bottom-up also added such comments. This means that just over half of the experts consider an alternative way to be the best choice for organising a formal methods course.

As could be expected from our suggestion in the option, a majority of 36 comments came from experts who opted for an alternative way. Nine comments indeed favour a combination of the two extremes, but 11 comments also mention that the answer depends on factors, such as the context, the lecturer, the course ("foundational courses [...] can be top-down; more applied courses [...] should be bottomup"), and the students ("bottom-up at the BSc level, [as] young students want to solve problems [...], top-down at the MSc level, [as] more mature students like to learn new theories [...]"). A few comments suggest to take inspiration from how we teach mathematics and programming.

From the comments, one can distil a fundamental motivation for the bottom-up approach, namely: we need to teach students to understand the problem and the requirements before selecting a particular tool or solution. More than half of the 13 comments from experts opting for bottom-up agree that "starting from examples is important", since "a good theory always comes with a good practical motivation". Six of the experts opting for an alternative way also mention a need for appealing running examples and non-trivial applications. Ironically, the only comment received by an expert opting for top-down is: "no good tools without theory!"

\subsection{Tool Usage}

This question asked the experts whether, and to which extent, students should be exposed to software tools when being taught formal methods.

\begin{tabular}{|l|r|}
\hline Which role should software tools play in formal-methods courses? \\
\hline \hline No role at all, as they obscure or divert from theoretical concepts & $0.0 \%$ \\
\hline $\begin{array}{l}\text { Marginal role: their existence should be mentioned to show that theoretical conc- } \\
\text { epts can be implemented }\end{array}$ & $3.1 \%$ \\
\hline Fair role: students should be told to learn by themselves about a few selected tools & $19.2 \%$ \\
\hline Major role: lab exercises on concrete applications should be assigned to students & $75.4 \%$ \\
\hline No opinion & $2.3 \%$ \\
\hline
\end{tabular}


An overwhelming majority of answers judged the use of tools essential when teaching formal methods. Moreover, nobody supported the idea that tools should be kept away from formal-methods lectures; this is one of the very few questions where one of the proposed answers has been chosen by none of the respondents. This confirms a high consensus about the usefulness of tools in teaching.

This question received 55 comments distributed as follows: 0 for no role at all, 3 for marginal role, 10 for fair role, 39 for major role, and 3 for no opinion. Most comments claim that tools "need to be integral part of the courses", since "many students enjoy working with tools", "exercises on paper are not convincing", "without tools you will not be able to convince students that things are applicable, i.e. in their minds: worth studying", and "unless students are able to apply the concepts they learn to concrete examples, theory will not stick". Other comments put forward that "if you want people to use tools, you have to get students to use some", because "if students get a feel of formal methods tools, they are more likely to apply them in practice during their professional life". Thus, "hands-on courses are needed" and "not only lab exercises but also almost all homework should involve tools". One comment suggests that "another option is to have students implement tools".

A few comments express various reservations: (i) tools should be carefully chosen, because "if students negatively perceive a tool, this also reflects on the applicability and usefulness of the theory"; (ii) tools are only part of a larger problem, as "formulating a good model is a huge challenge in many cases" and students should "understand what the tools are saving them from having to do themselves"; (iii) there should be a correct balance between theory and practice, as "theoretical aspects [are] not always taught best with tools only" - a comment notices that "this is the same as the debate about teaching things that can be used immediately vs. teaching foundations that will be valid in 25 years from now".

\section{$7 \quad$ Future of Formal Methods}

\subsection{Future Dissemination}

This first question on the future of formal methods aimed to evaluate the long-term industrial uptake of formal methods.

Do you believe that formal methods will eventually spread more widely in industry? \begin{tabular}{|l|l|l|l|c|}
\hline Definitely: 37.7\% & Probably: 52.3\% & Probably not: 6.9\% & Definitely not: 0.0\% & N/A: 3.1\% \\
\hline
\end{tabular}

A huge majority of $90 \%$ thinks the use of formal methods will likely become more widespread in industry, while only nine experts doubt this and four have no opinion.

This question received 57 comments. From the experts who doubted an increasing use of formal methods in industry, two comments base their expectation on what happened in the past; one comment thinks that "industry is just too conservative for that"; one comment urges the formal methods community to "radically change the way we 'sell' formal methods"; and one comment displays a general disappointment with society, concluding that "it does not look like that the humankind will be interested in software quality". 
Fifteen comments justify an increase of the application of formal methods by an expectation for a growing demand, either because "the risk induced by (cyberphysical) systems [...] will be omnipresent", or because "with the increased advent of certification regulations, industry companies having their products certified will have a competitive advantage". Several comments mention the growing complexity of systems, which "get so complex and hardly predictable that they need all type of computer-aided support to assure safety and correct functioning". One comment identifies a new mission for formal methods: "because [unmanned, autonomous] systems cannot rely on a human operator to act when a serious problem occurs, developers of autonomous systems want high assurance that these systems behave safely and securely and that they are functionally correct".

A few comments believe that the tools will become easier to use in the future. Yet, successful technology transfers might remain confidential, since a comment reports that "once a formal method tool achieves success, it is usually given a new name, probably to avoid the stigma of being a 'formal method'”. Finally, another comment expresses careful optimism: "the word is getting out! More companies are hiring formal methods engineers".

\subsection{Future Users}

This question aimed at predicting the target audience for the future applications of formal methods.

\begin{tabular}{|l|l|}
\hline Who are most likely to use formal methods in the future? \\
\hline \hline A large number of mainstream software engineers & $42.3 \%$ \\
\hline A small number of skilled experts & $43.8 \%$ \\
\hline Others & $13.8 \%$ \\
\hline
\end{tabular}

The experts were quite divided on this question, since the first two proposed answers attracted nearly the same number of proponents (55 vs. 57 experts).

The analysis of the 65 comments received provides more insight. From the 18 experts who selected others, actually 12 indicate in the comments that they believe that the answer is both: "a large number will make small, rote usage of the tools, [while] a small number of skilled experts will be heavy users".

Two comments express the belief that domain experts will be the power users of formal methods. One comment wonders how hardware experts fit in the question, but another comment confirms their role: "within hardware development, it has been standard practice to perform Logical Equivalence Checks for some time; this is a specialised use of SAT to check equivalence of two circuits and it is used for translation validation and sometimes to check manual optimisations".

Many comments further distinguish between various kinds of formal methods. Mainstream software engineers are expected to use lightweight formal methods, particularly the automated ones, which are hidden in standard development tools. This idea is present in at least 22 comments. On the other hand, specialists will always be needed to advance tool development; several comments also claim that the explicit use of formal methods will require experts, whenever skills like modelling, specification, abstraction, and interactive proof generation are involved. 
It is encouraging that many experts envision a wide audience of mainstream software engineers as future users of formal methods. The fact that this is still not happening is attributed by some to the low quality of (automated) tools: "until enough progress is made to make formal methods accessible to mainstream programmers, only trained experts will be able to use the tools". Others explain the issue by a lack of appropriate scientific/technical education.

\subsection{Promising Applications}

This question tried to list all domains in which formal methods may have impact.

\begin{tabular}{|l|r|}
\hline Do you foresee promising upcoming applications of formal methods? \\
\hline \hline In other branches of computer science & $69.2 \%$ \\
\hline In finance (digital currencies, smart contracts, etc.) & $61.5 \%$ \\
\hline In other sciences (biology, etc.) & $58.5 \%$ \\
\hline In politics (e-voting, e-government, etc.) & $43.8 \%$ \\
\hline In other parts of society & $31.5 \%$ \\
\hline
\end{tabular}

(multiple answers allowed; answers sorted by frequency)

The experts appeared rather optimistic (60-70\%) concerning new applications of formal methods in hard sciences (including biology), but less convinced (30-40\%, which is still important) by applications in social/human sciences and other parts of society. A pessimistic expert added one missing option: "I see them nowhere".

The question attracted 144 comments. Many of them explicitly suggest applications in other branches of computer science, among which (i) software engineering: program synthesis, legacy software, aspects, product lines, human interfaces, business process modelling, etc.; (ii) networking and distributed systems: internet of things, sensor networks, security, etc. (iii) safety-critical systems: embedded systems, robotics, cyber-physical systems, control software for infrastructural systems, etc.; (iv) data science, machine learning, and artificial intelligence; and (v) traditional fields such as compilers, databases, algorithms, numerical computing. As one comment observes: "all those communities realise their problems are too hard to solve just by brute force, and growing calls for reliability in these fields are forcing investigation into and adoption of formal methods".

Among other sciences, the most frequently cited ones are biology, epidemiology, surgery, and medicine: "I am excited by the work in model checking of biological systems! Maybe we can even help with drug development?" Chemistry and physics are also mentioned ("one can perfectly well see a hydrogen atom as a state machine"), as well as engineering disciplines such as automotive, transportation, traffic control, aerospace, power or energy control, and the verification of numerical simulations. Social sciences, including law, are also considered as potential application areas.

The comments are optimistic about applications in finance, in particular to make transactions more secure. One comment phrases the urgency as: "we sit on a financial bomb". This raises many interesting challenges: "highly complicated cryptography and contracts need formalism". Several comments point to recent successes in formalising blockchains and smart contracts. There are also grander visions, such as 
developing "formal models of the entire financial service industry, banks, [and] stock exchanges".

Several comments mention that more research on e-voting is required, since "trust is really required in that domain". As a comment predicts, "formal methods will show that we have to be careful with e-voting". More ambitious expectations consider a much larger scope: "Perhaps more formalisation of laws and regulations" is needed, and "I would like to see formalised notions of fairness, causality, justice, etc.". Finally, the comments addressing in other parts of society largely overlap with the aforementioned ones.

\subsection{Potential Competitors}

This question polled the experts whether other rising approaches that are increasingly in competition with formal methods to get research funding, to capture industry interest, and to attract students might eventually overshadow formal methods.

Do you believe that alternative approaches (e.g. artificial intelligence or quantum computing) will eventually replace formal methods?

\begin{tabular}{|l|l|l|l|l|}
\hline Definitely: $0.0 \%$ & Probably: 3.1\% & Probably not: 41.5\% & Definitely not: 51.5\% & N/A: 3.8\% \\
\hline
\end{tabular}

A vast majority (93\%) of the experts stated that formal methods will (probably or definitely) not be replaced by alternative methods. No expert believed that this will definitely happen. Five experts indicated they have no opinion on this matter.

Analysing the 72 comments received, 26 of them indicate that formal methods are incomparable or complementary to the proposed alternatives. A few comments criticise the question for this reason. Interestingly, 38 comments stress that formal methods and artificial intelligence can strengthen each other. Only 10 such remarks were made for quantum computing. No comment mentions another alternative than the two proposed ones.

Several comments explain why neither artificial intelligence nor quantum computing can replace formal methods. Many of them argue that only formal methods can provide guarantees about correctness, e.g. "artificial intelligence is wrong in 10 to $25 \%$ of the cases and must be hand-tuned. What is formal there?" and "artificial intelligence will need to be certified. What methods will be used to certify it?".

A quite different reason is provided in two comments that praise the crucial role of formal methods in requirements specification: "at the end of the day, both the ambiguous setting and the mapping to the unambiguous setting are characteristic of human activities. I have a hard time imagining that these creative aspects can be fully automated".

Other comments see fruitful interactions between both fields. Formal methods may help understand artificial intelligence, generate explanations, assist the certification of machine-learned components, or complement them with safety supervisors. In turn, artificial intelligence can improve formal methods by providing heuristics for guiding proof search: "I have seen artificial-intelligence-guided first-order provers that can learn from manual interactive proofs". Another comment adds: "we need approaches that combine model-driven and data-driven techniques". 
Although less frequent, similar comments appear for quantum computing: "of course, quantum computing could provide a big hammer", but "there will still be a place for formal methods to study the computational models and perform reasoning about quantum computing" and "formal methods have also been investigated to show correctness of quantum programs".

Finally, we mention a few diverging opinions. Two comments recall that certain parts of formal methods (in particular, symbolic reasoning) were originally a branch of artificial intelligence. Two other comments fear that, in the perception of the public, artificial intelligence could replace formal methods: "but in terms of 'branding', formal methods might disappear from the perception of users who may think of these things more as artificial intelligence. This will require active intervention".

\subsection{Major Breakthroughs}

This last question on the future of formal methods wanted to know whether a scientific breakthrough can be expected any time soon.

\begin{tabular}{|l|l|l|}
\hline $\begin{array}{l}\text { Do you expect that a major breakthrough ("game changer") } \\
\text { will happen in formal methods? }\end{array}$ \\
\hline Not really: $33.8 \%$ & Within 2 years: $0.0 \%$ & Within 5 years: $12.3 \%$ \\
\hline Within 10 years: $17.7 \%$ & Within 25 years: $6.9 \%$ & N/A: \\
\hline
\end{tabular}

The answers listed in this table are better understood by examining the 46 comments received for this question. We first discuss those comments arguing that a breakthrough is not really expected. Ten comments foresee a more gradual, evolutionary progress of the techniques tending to their widespread adoption. Five comments point to external factors, e.g. "societal and economic factors" and "the cultural barrier to the use of formal methods". Another comment shows some hope: "the only game changer I would see, if more and more standards, certifications, and regulations demand the kind of guarantees that only formal methods can provide".

From the comments that, sooner or later, expect a breakthrough, three of them see it coming from killer applications, six others from a particular combination of methods, and four others from a single technical development that could be a game changer. Interestingly, the advances in SAT/SMT solvers are mentioned, in those three sets of comments, as the example of the most recent game changer.

An example of such a potentially groundbreaking combination is given: "just as tools (e.g. solvers) in formal methods have grown tremendously over the past few decades, so too have tools in other areas: most obviously in machine learning, but also in fields like stochastic optimisation. Putting together all these in meaningful ways may lead to dramatic improvements in all of them".

Three examples of individual advances that could lead to a breakthrough are also given: "synthesis of correct-by-construction control components for critical systems", "serious use of models instead of programs, coupled with automatic generation of code", and applications of "big data" or "quantum computing". 


\section{Conclusion}

Formal methods are now more than 50 years old, and after half a century of sustained research, development of new techniques, and continuous enlargement of the perimeter of formal methods, it was high time to review the situation: the 25th anniversary of the FMICS conference was a suitable opportunity to do so.

The present survey is an unprecedented effort to gather the collective knowledge of the formal methods community. Not less than 130 internationally renowned experts agreed to participate in the survey and spent significant time to express their views, through answers to our questionnaire, through detailed comments accompanying these answers, and through position statements that deliver the personal opinions of these key actors in formal methods.

Many lessons can be learned from all these contributions, the collective ones are synthesised in Sections 3-7, while the individual ones can be found in Section 9. The general opinion is that formal methods achieve many technical successes, but are not yet mainstream to their full potential. There is still much to be done and, among all the pending tasks, we wish to highlight three action points more particularly:

- The results of the survey indicate a consensus about the essential role of education to give the next generations of students a sufficient background and practical experience in formal methods. Unfortunately, it appears that the current situation is very heterogeneous across universities, and many experts call for a standardisation of university curricula with respect to formal methods. A recent white paper [7] provides a good starting point for such an undertaking. Also, one should not neglect continuing education and make sure that industry professionals who did not attend university classes can learn about formal methods from alternative channels (online courses, tutorial videos, etc.).

- The results of the survey also make it clear that formal methods are no longer a paper-and-pencil activity: like other fields such as logic and computer algebra, formal methods have shifted their orientation, and their progress now closely relies on software tools. A majority of experts considers that universities and research institutes have a central role to play in the construction of such tools. However, software development is often underrated by standard academic evaluation, which primarily measures excellence in terms of publications in scientific conferences and journals. Thus, many experts call for a revision of the current academic reward system to better encourage long-term investment in the development of innovative, high-quality software. Researchers are also invited to join forces to build common platforms that can become part of mainstream development practices.

- Computing takes an ever-growing importance in modern societies but is still much less regulated than other sectors (transportation, real estate, healthcare, etc.), even though software or hardware bugs may have dire consequences in an increasingly connected digital world. The industrial dissemination of formal methods really progresses when companies that produce software or softwareintensive systems decide to protect the safety, security, and privacy of their customers - thereby protecting their own assets and reputation at the same time. 
The current incentives for such virtuous behaviour are probably not enough, and many experts call for a greater regulation of software quality (beyond the traditionally supervised sectors of aerospace, nuclear energy, and railways), with stricter standards that examine the final product rather than its development process, and a stronger promotion of best practices by professional bodies. Such measures (together with, e.g. finer risk assessment of software products by insurance companies) would turn formal methods into a profitable investment.

We hope that this survey will highlight the potential of formal methods to the numerous stakeholders (policy makers, regulators, company managers, research funding agencies, professionals, students, etc.) and encourage industry to use these methods more intensively.

\section{Position Statements}

Each expert who answered all 30 questions of the survey was then warmly invited (yet not required) to write a short statement (not exceeding 10 lines) about formal methods. Guidelines were given in the form of three questions, with the expectation that each position statement would address these questions, or a subset of them:

1. Personally, what do you consider to be the next challenges for formal methods?

2. How are you currently contributing to these efforts?

3. Which of your contributions could be most beneficially picked up and carried forward by the next generation?

It was stated that each position statement would be nominally attributed to its author, the intention being to confront individual visions from many high-profile experts - contrary to the 30 questions of the survey, whose answers would be anonymised to distil the collective opinion of the formal methods community.

Nearly 60 acronyms occur in the position statements. In below tables, we only expand those needed to understand ideas; we neither detail acronyms defined before being used in position statements, nor well-known acronyms (OS, PC), nor names of computer languages (UML, VDM), software tools (CADP, SPIN), universities (ECNU, RWTH), organisations (ISO, NASA), and conferences/workshops (FMICS, MARS).

\begin{tabular}{|c||l||c||l|}
\hline \multicolumn{1}{|l|}{ Acronym } & Signification & Acronym & Signification \\
\hline \hline AI & Artificial Intelligence & IT & Information Technology \\
\hline CPS & Cyber-Physical System & JSON & JavaScript Object Notation \\
\hline CTL & Computation Tree Logic & LTL & Linear Temporal Logic \\
\hline DSL & Domain-Specific Language & ML & Machine Learning \\
\hline FM(s) & Formal Method(s) & PLC & Programmable Logic Controller \\
\hline GPU & Graphics Processing Unit & PR & Public Relations \\
\hline GUI & Graphical User Interface & REST & Representational State Transfer \\
\hline HMI & Human-Machine Interface & SAT & Boolean Satisfiability Problem \\
\hline HW & Hardware & SMT & Satisfiability Modulo Theories \\
\hline IP & Intellectual Property & SW & Software \\
\hline
\end{tabular}

Please note that each position statement reflects only its authors' views, and not necessarily the opinions of the authors of this report, nor those of their employers. 
The scope of FMs extends beyond program and model verification. FMs are applied in Workflow and Business Process Management, and recently in data-driven approaches like process mining. This reconfirms the importance of concurrency theory, a subfield of FMs rooted in early work of Carl Adam Petri. Petri's guiding principle was "Concurrency should be a starting point for system design and analysis and not added as an afterthought (locality of actions)". Operational processes are inherently concurrent, and the availability of event data allows to discover such processes. I anticipate FMs and data science to converge. We need FMs to describe real-world phenomena, and the abundance of data offers a unique opportunity. Thus, the practical applicability of FMs will continue to increase. It is vital that students learn to abstract and structure, and FMs are the tool for this. Edsger Dijkstra once said "Beauty Is Our Business". I would like to add “... and business is good”.

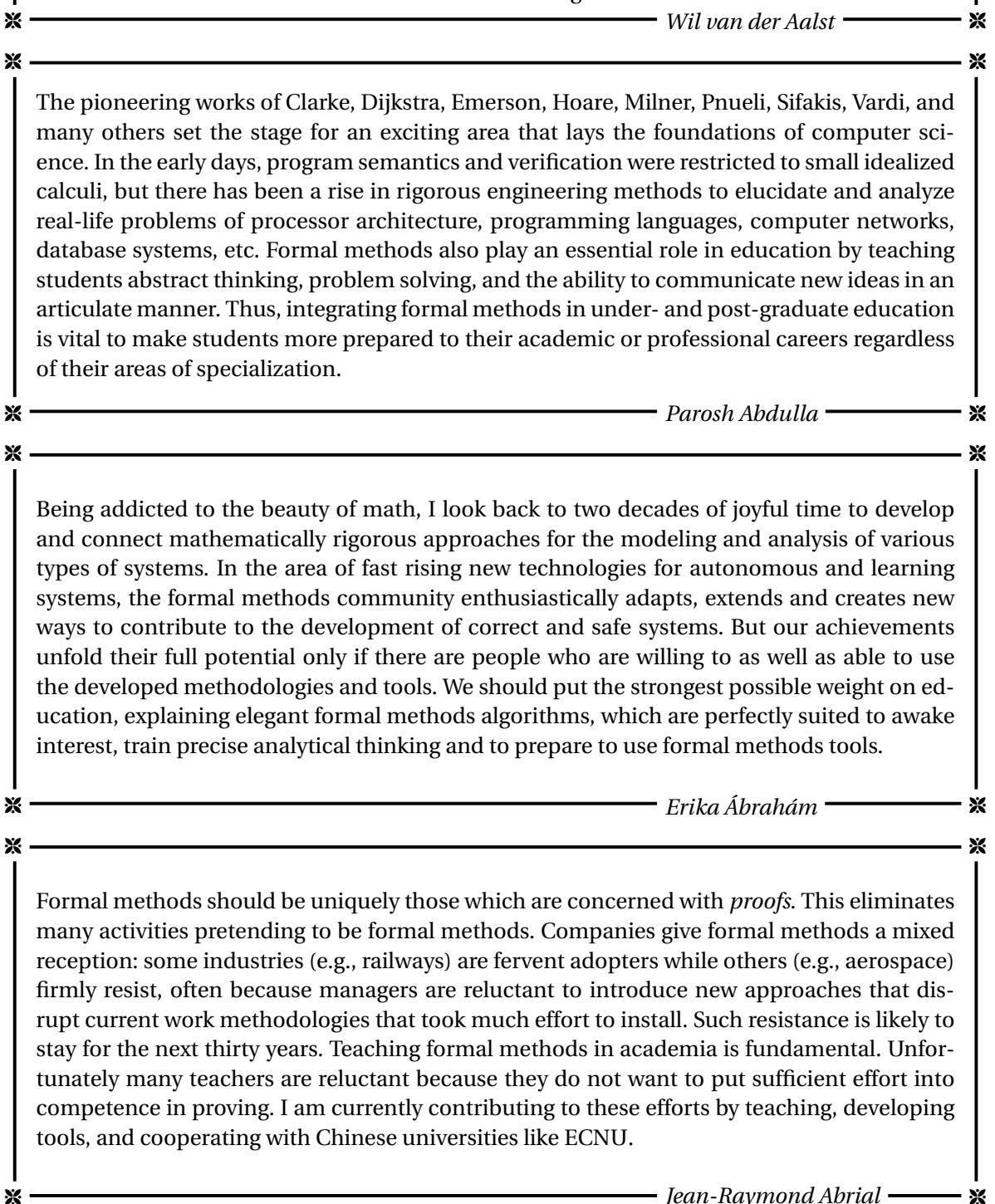




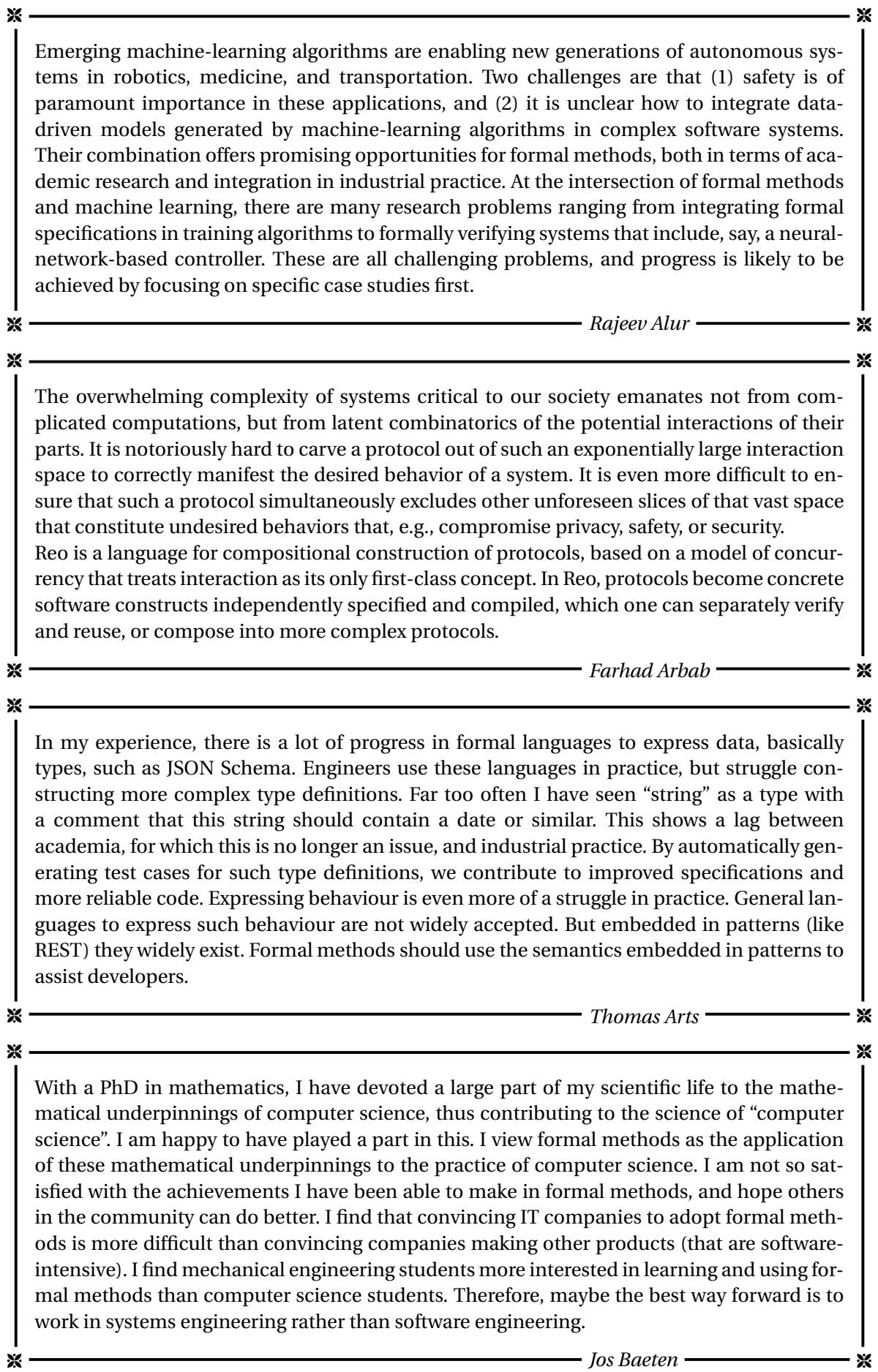




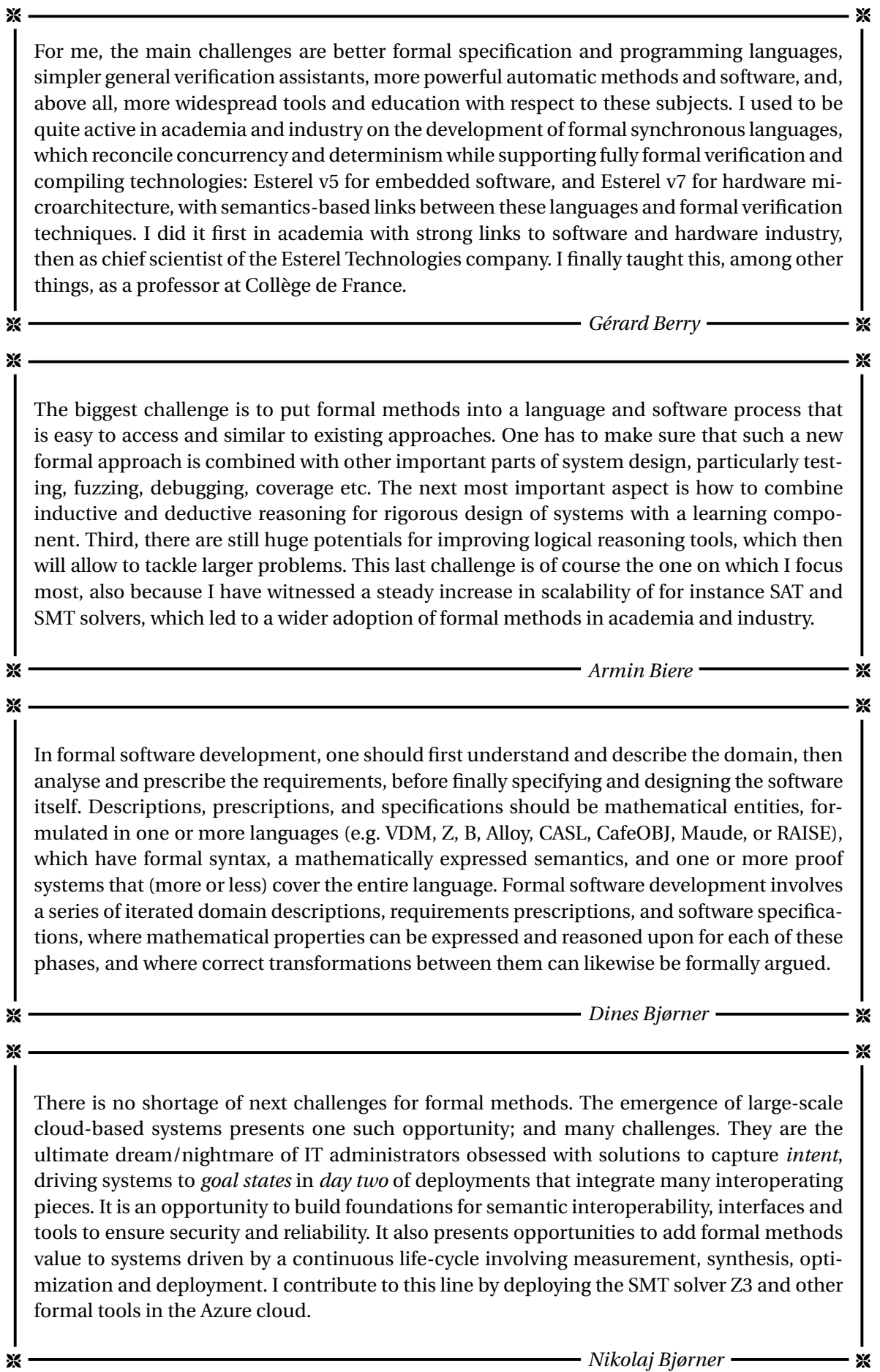


Recently, NASA looked for a "formal methods engineer". We should always present formal methods explicitly and consistently as an engineering discipline within computer science: "formal methods" alone is a too generic term. Underlining the engineering aspect in research and education clarifies their practical role. We should teach "FM engineering" as a specialisation of software engineering. FM engineering applies formal methods to improve software reliability, but also involves software development to support the application of formal methods. Underlining the engineering aspect in research would counter-balance the limited understanding of formal methods as a purely theoretical research topic. Hopefully, underlining the engineering aspect will help promote formal methods in academy and industry, and the term "formal methods (software) engineering" will broaden the understanding and appreciation of formal methods.

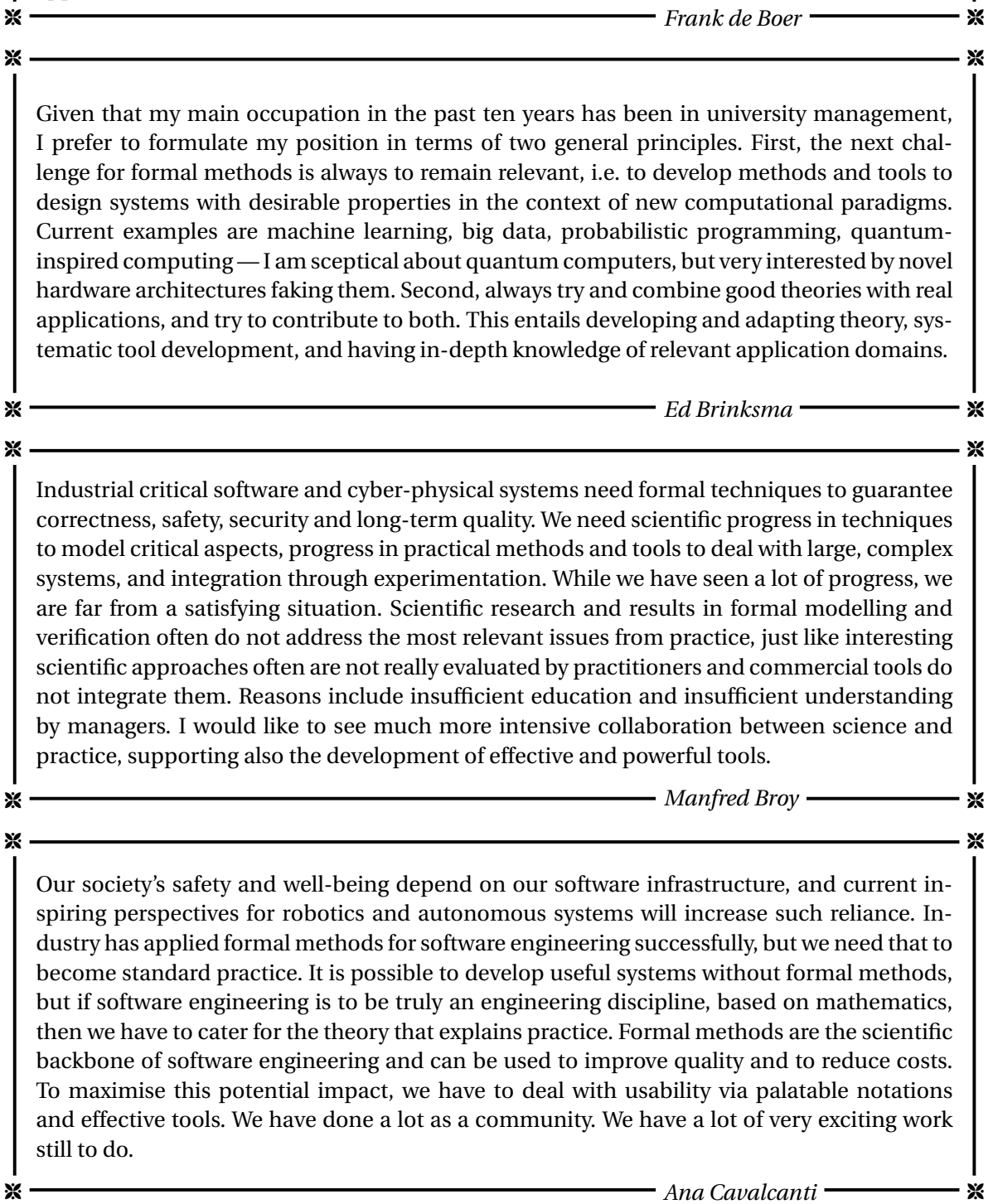




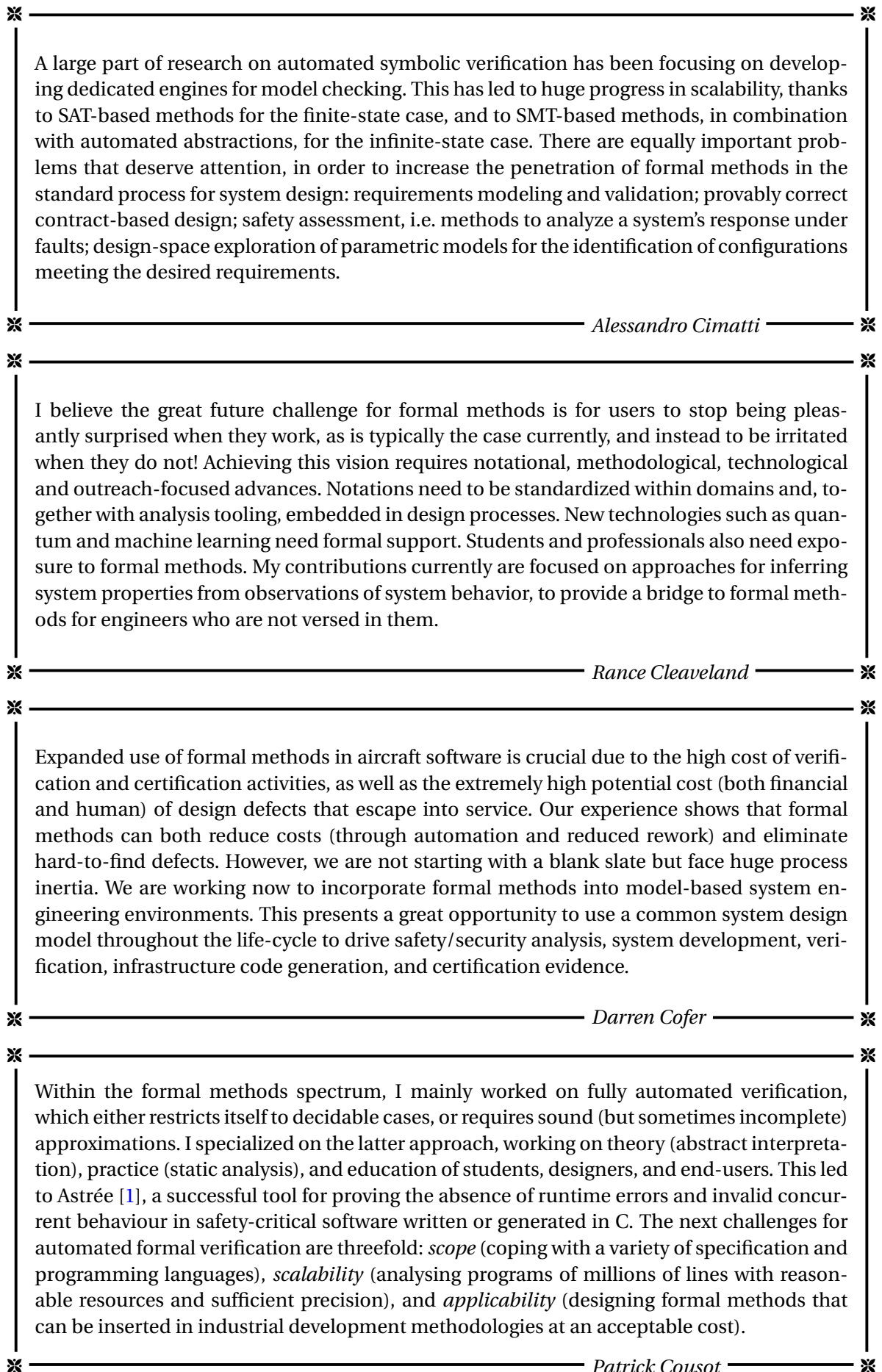




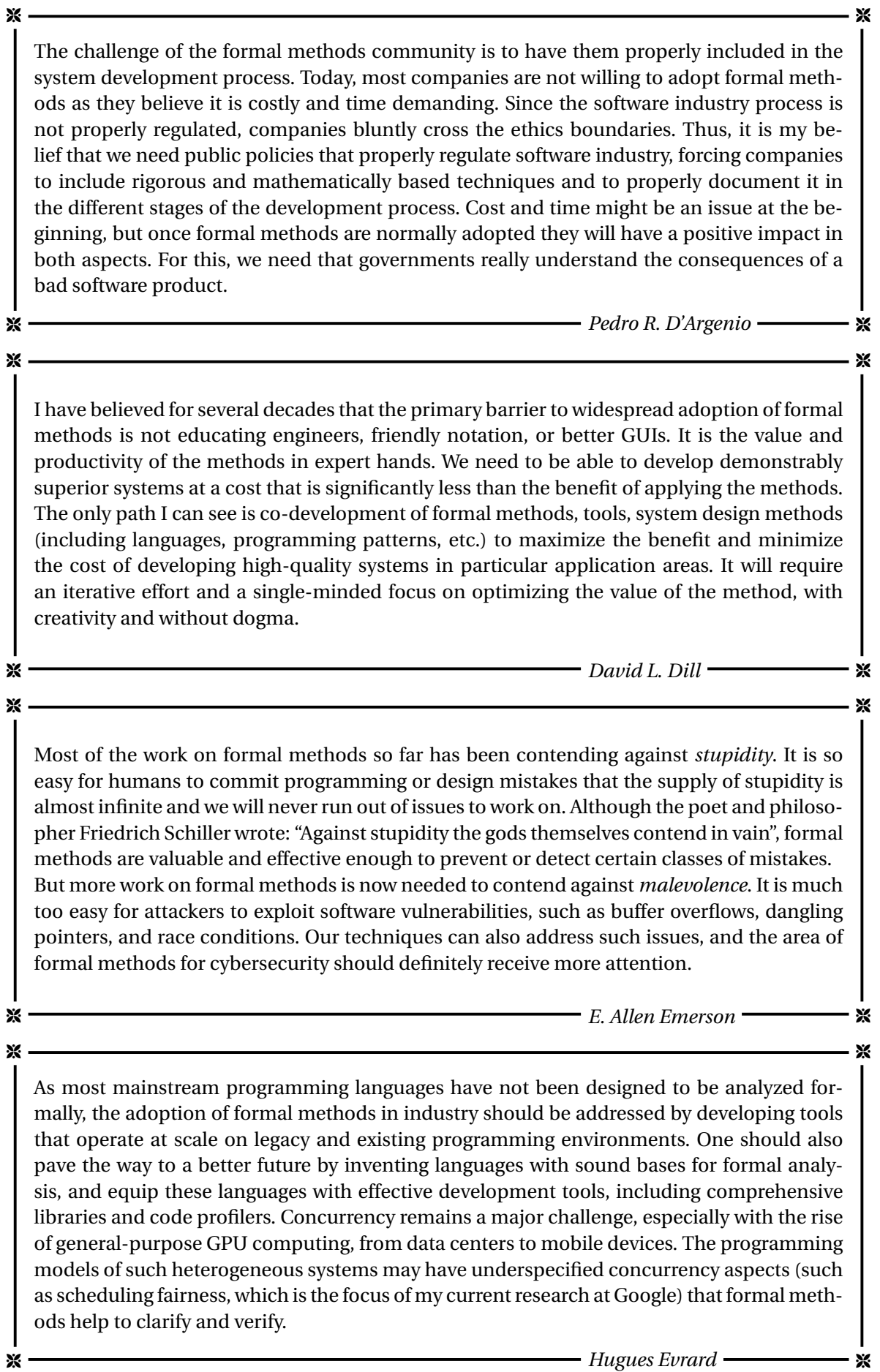




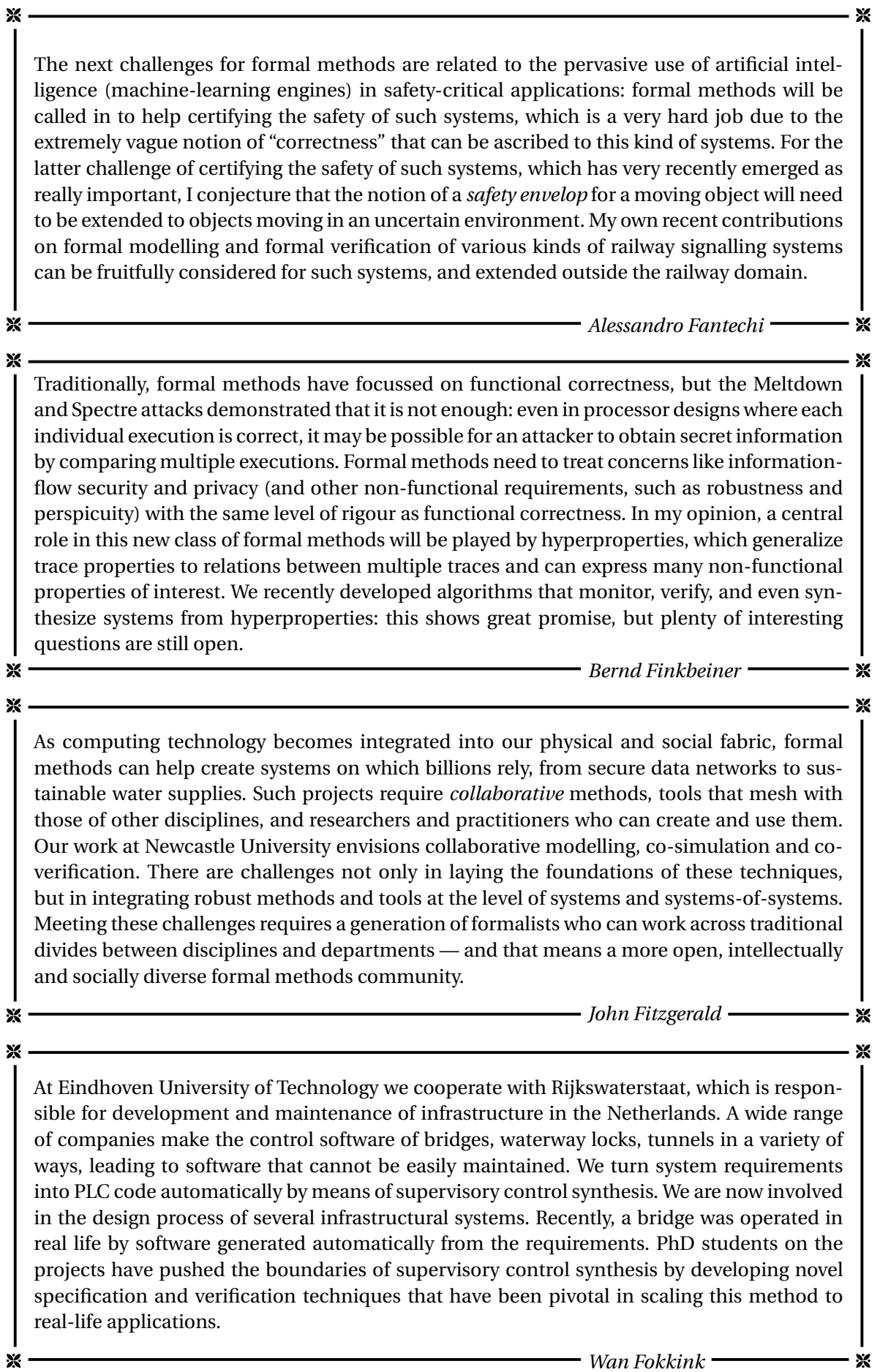




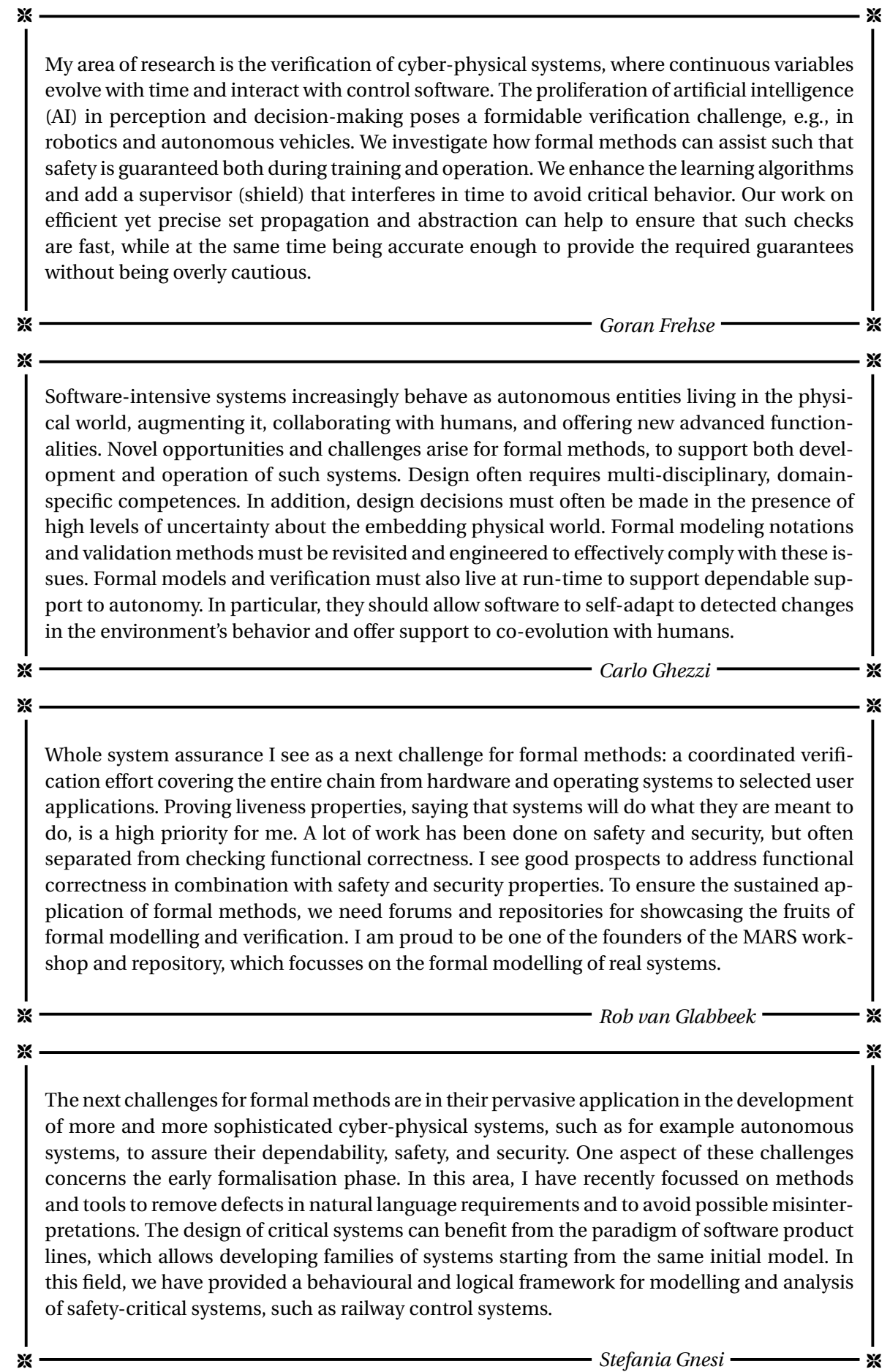




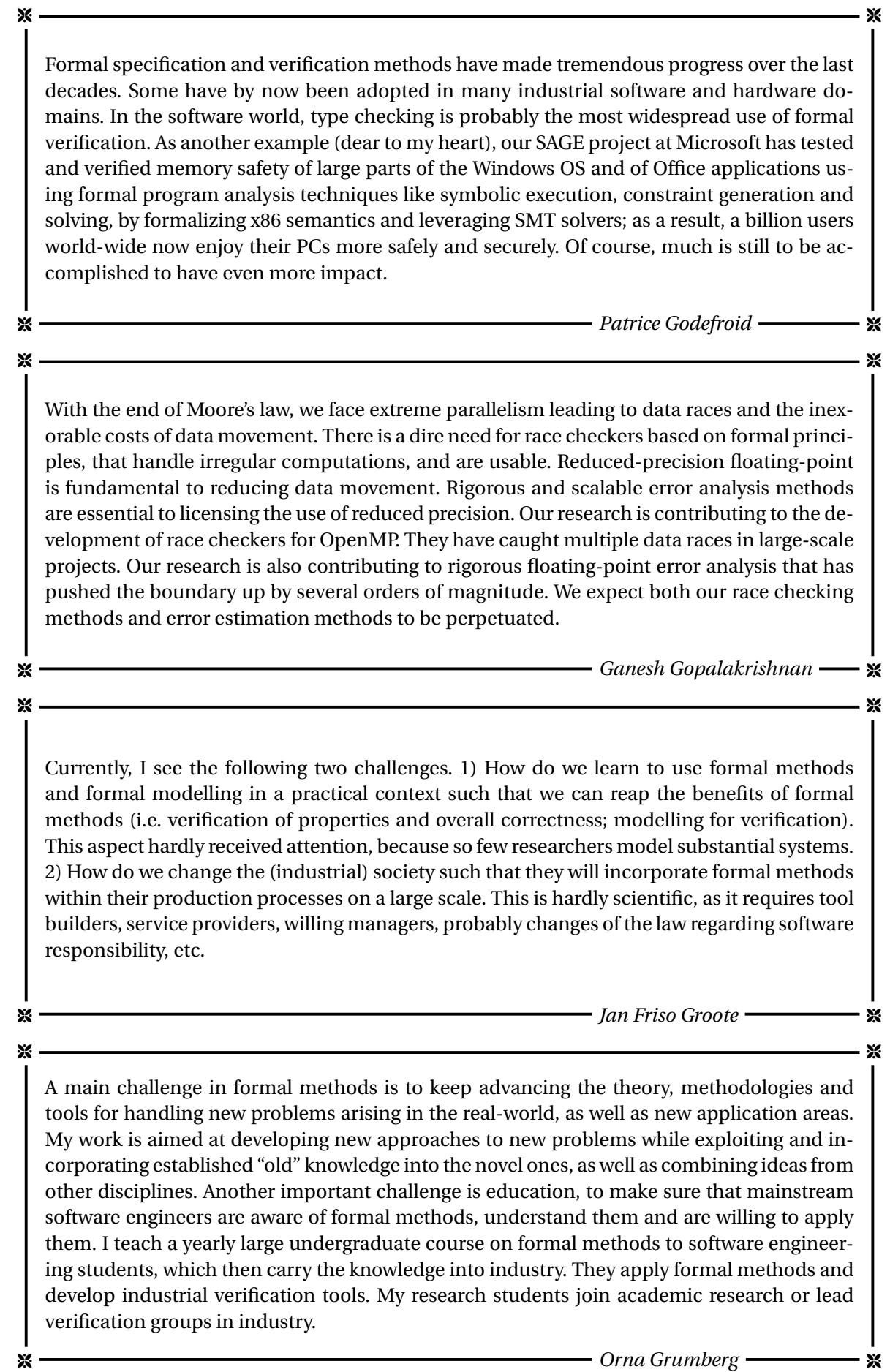




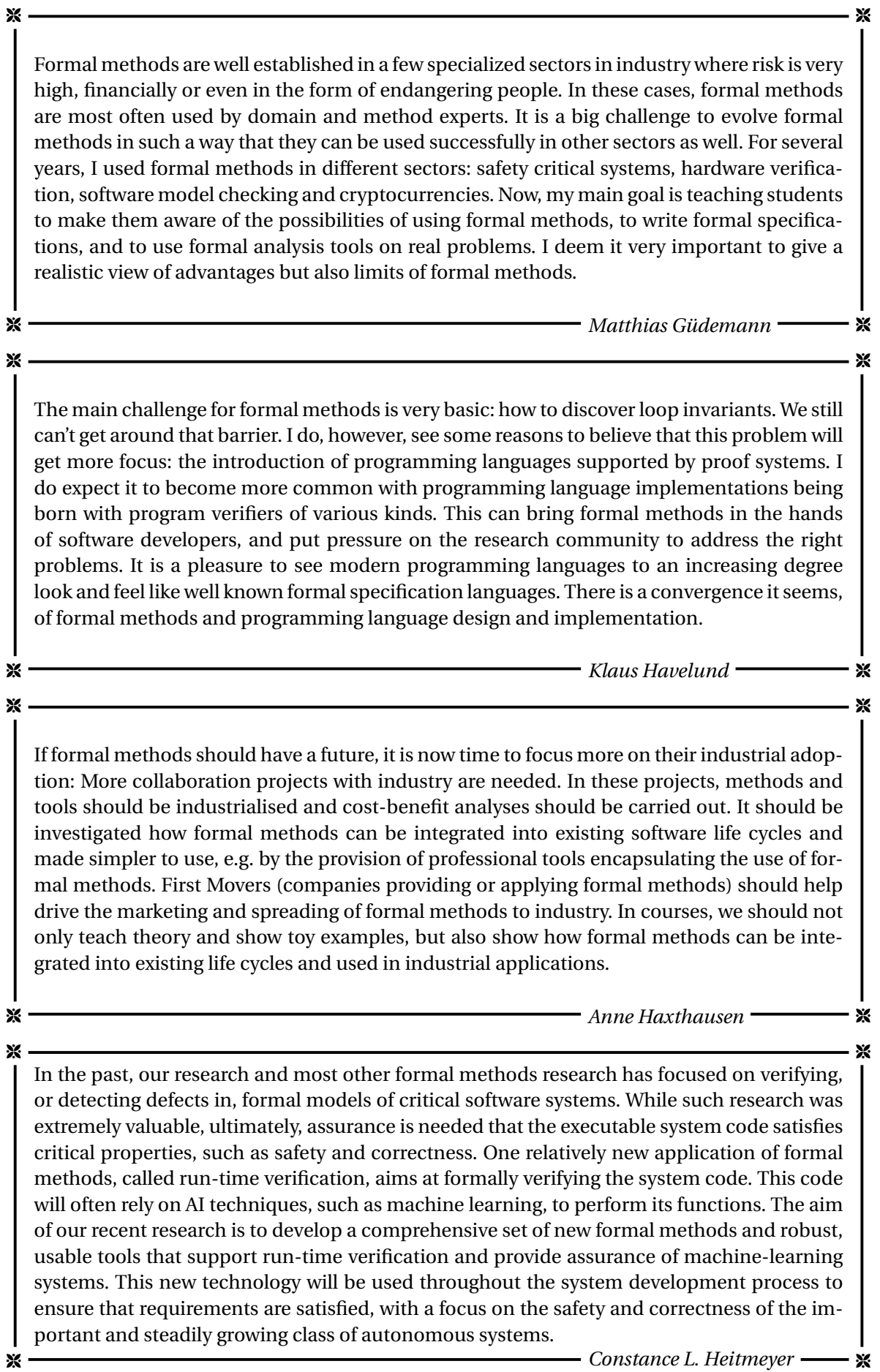




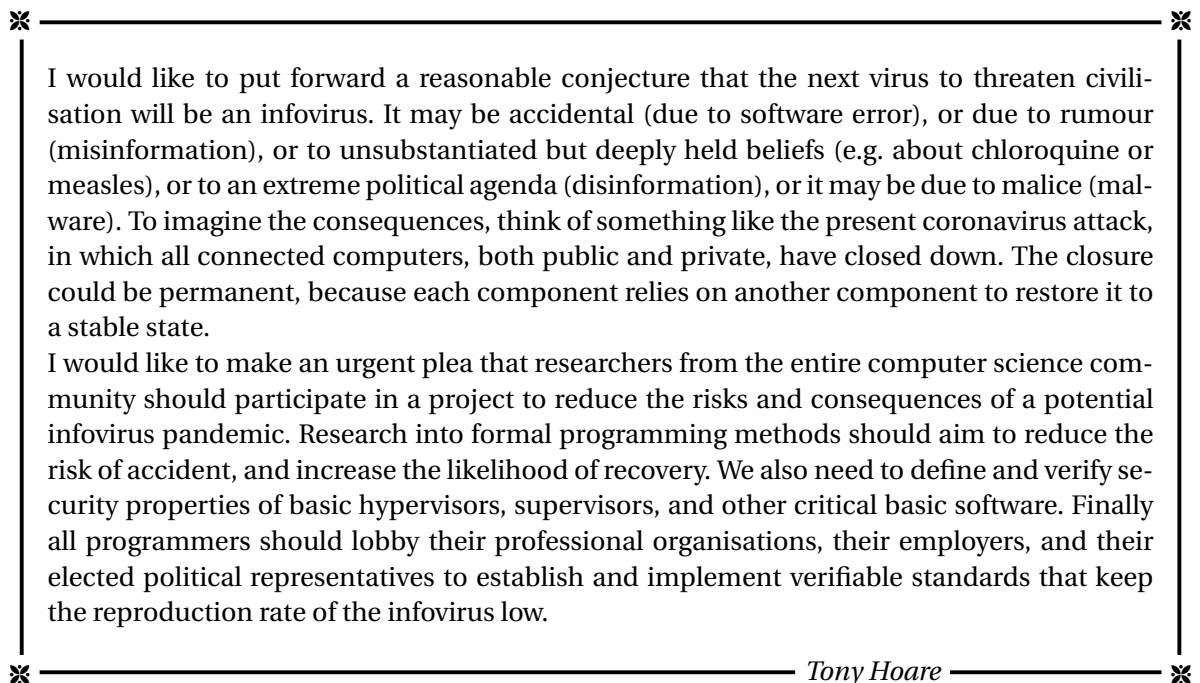

Tony Hoare

I believe that there is considerable benefit in the further development of formal methods
based tools that can perform analyses in real-time, interactively. In my own work, I have tried
to develop two types of methods that can achieve this. The first concerns a swarm verification
strategy that is aimed at large cloud computing environments, where we launch large num-
bers (up to millions) of very small, and very quickly executing search engines in the cloud, to
jointly deliver a verification result with high confidence of full coverage of a complex prob-
lem domain. The second concerns the development of the Cobra tool (github/nimble-code)
to perform interactive static analysis on large code archives, including, most recently, new
analyses for cybersecurity vulnerabilities. More research in this area of interactive formal
analysis is needed.

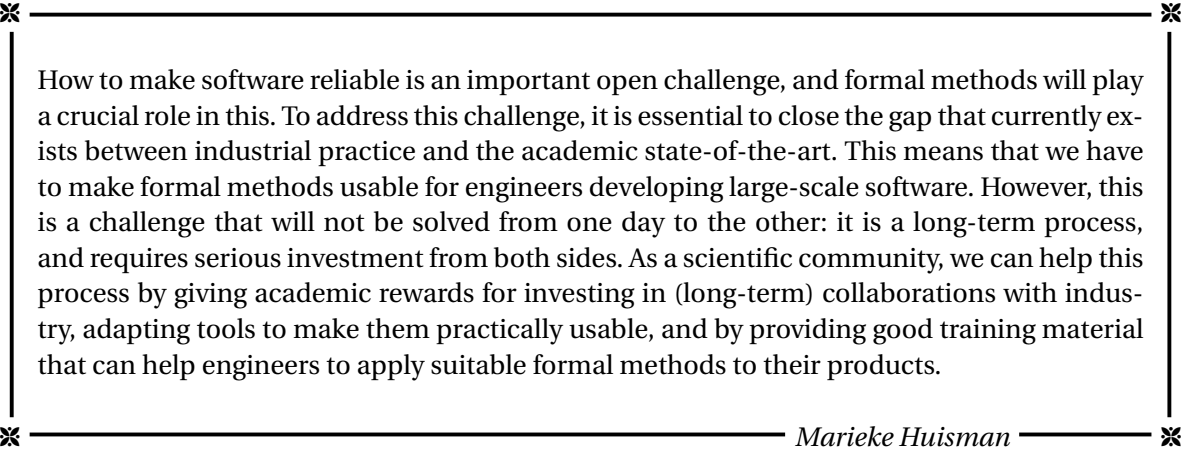




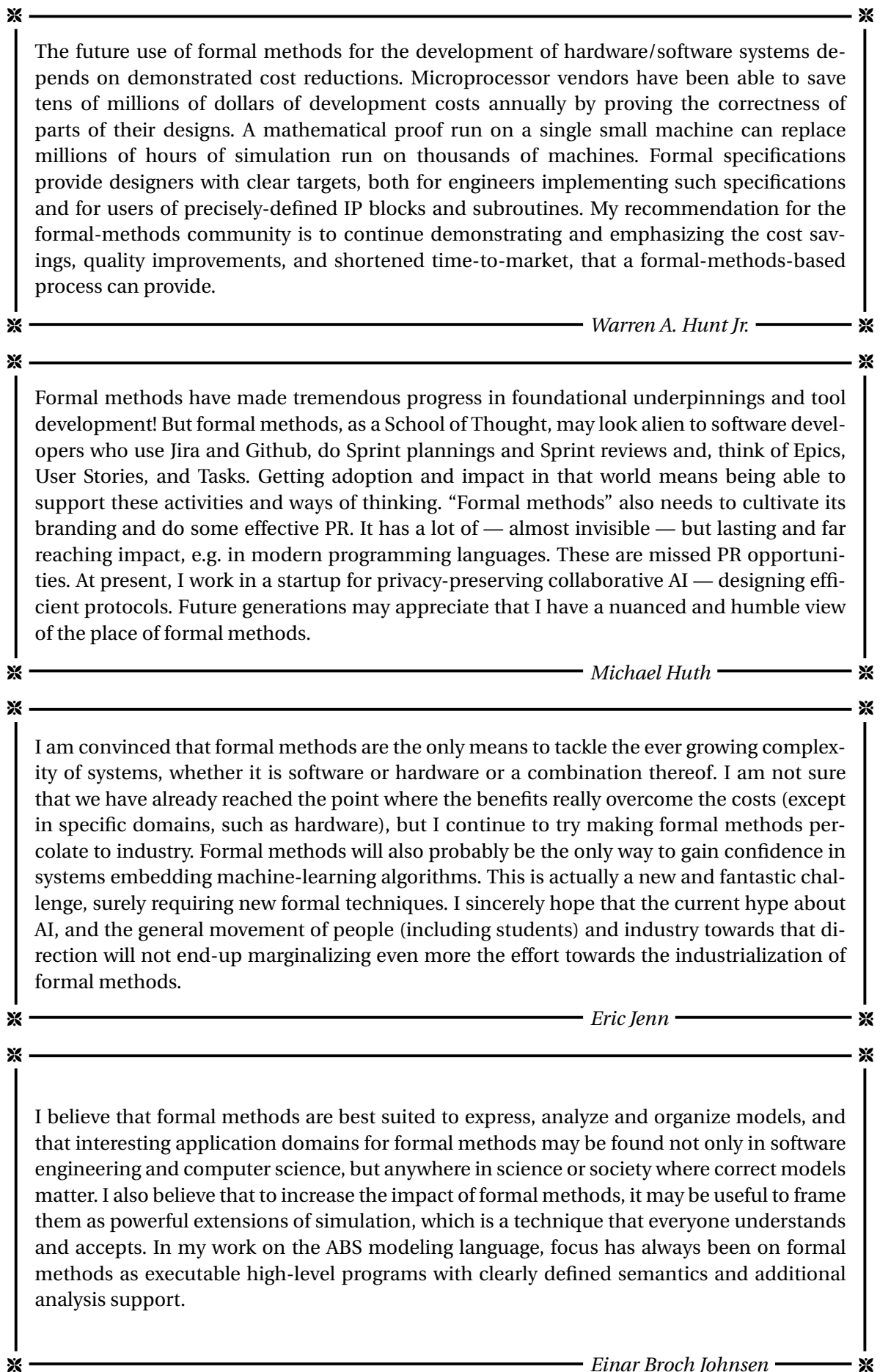




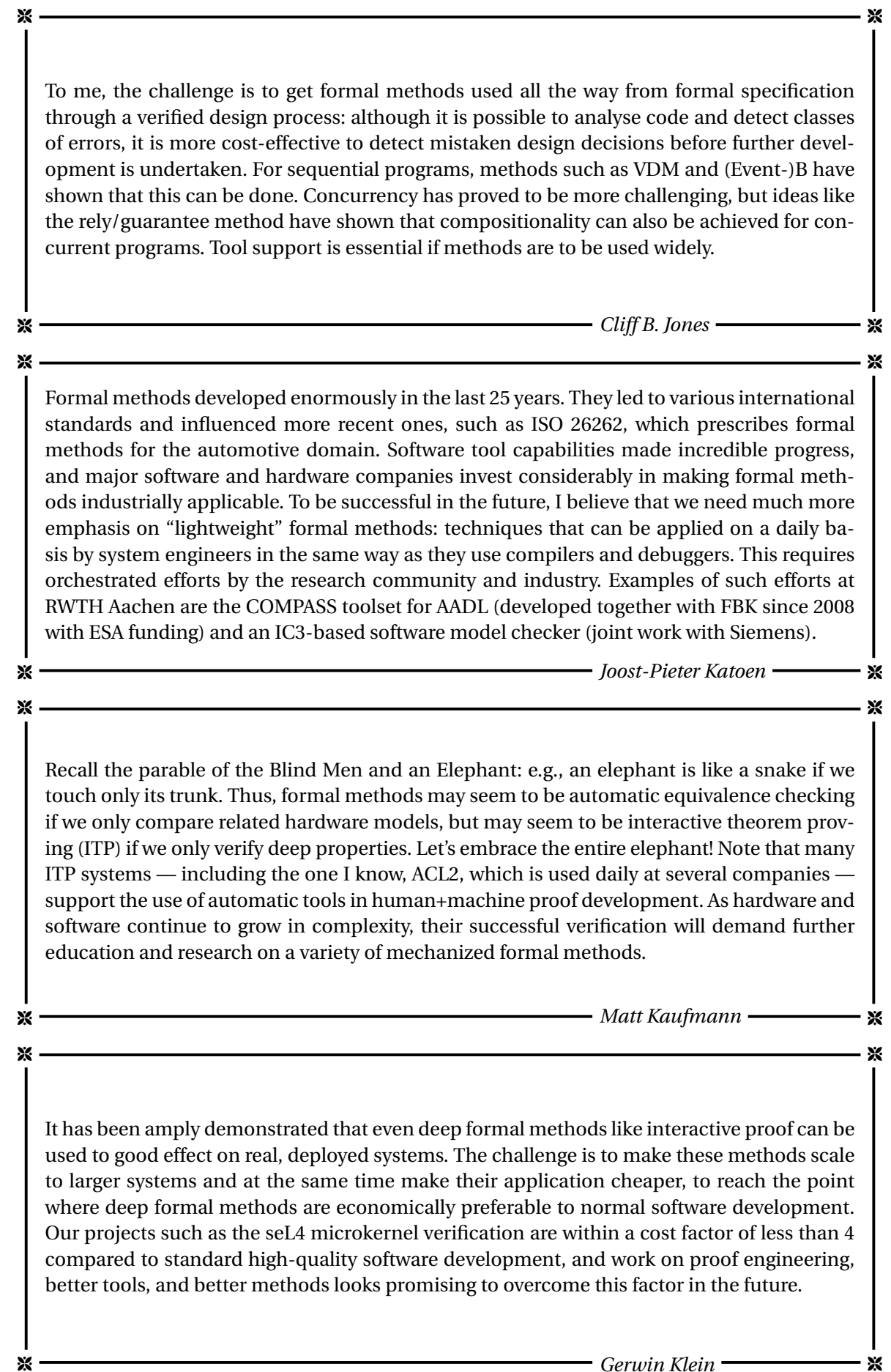




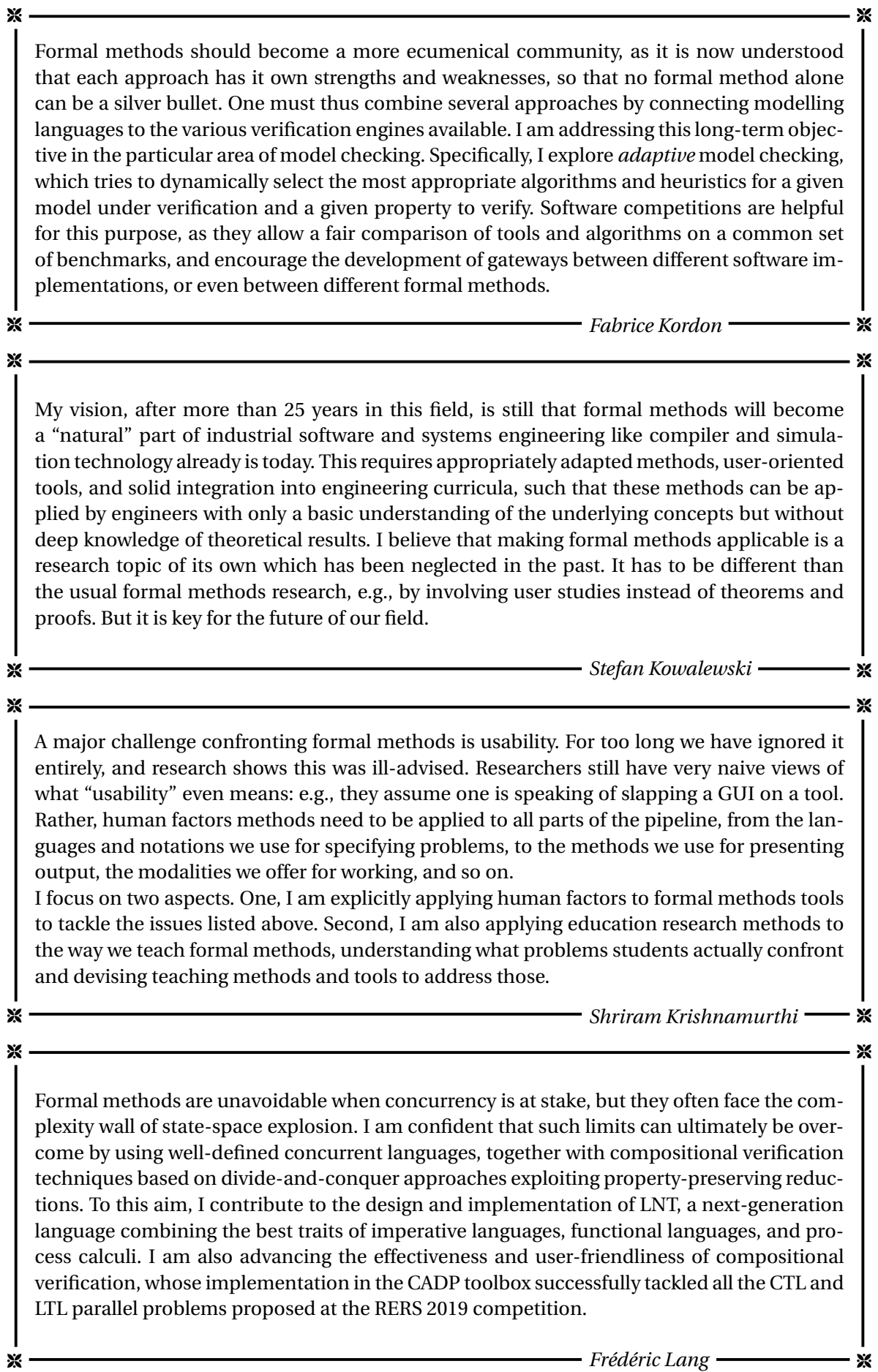




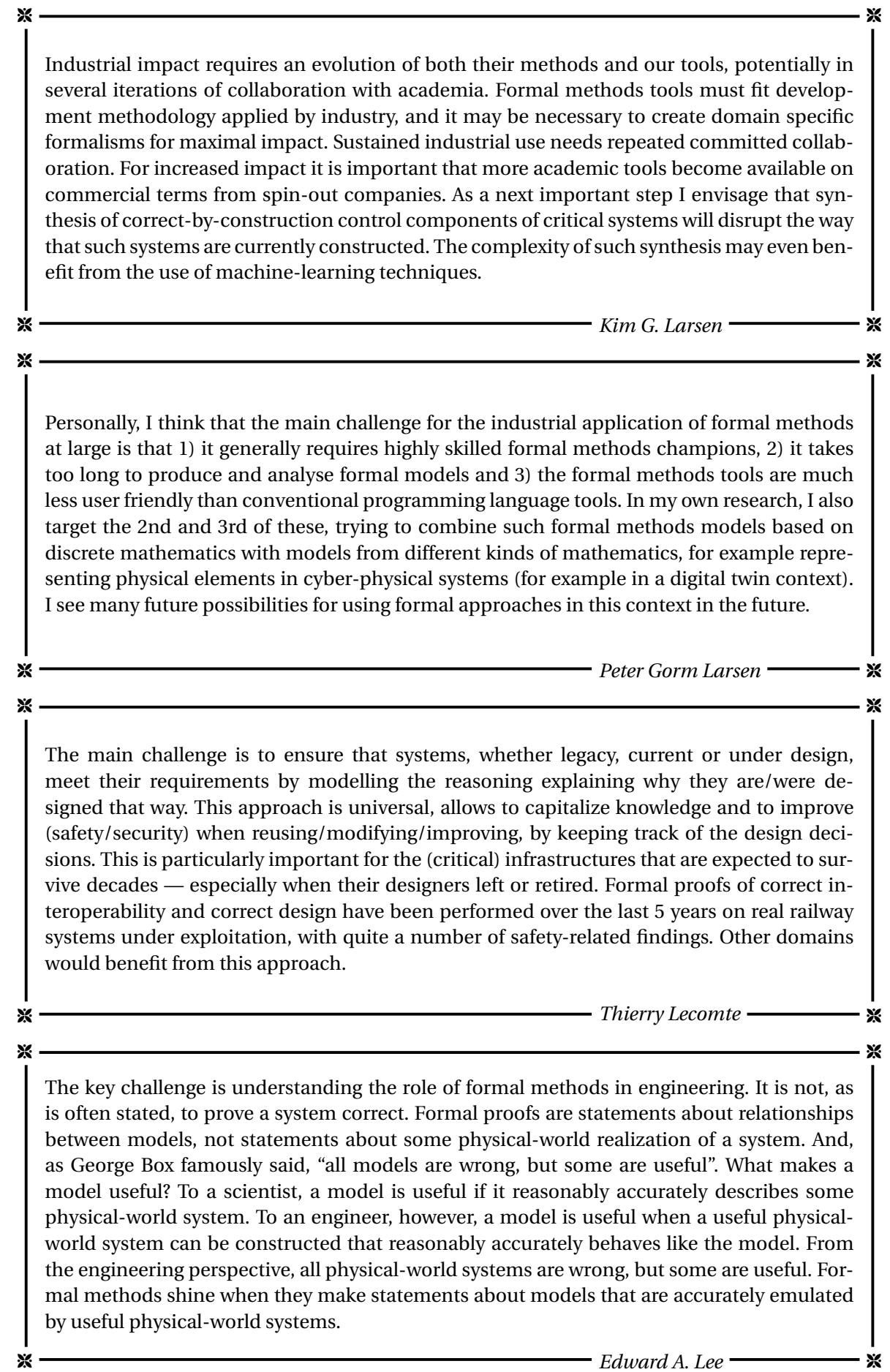




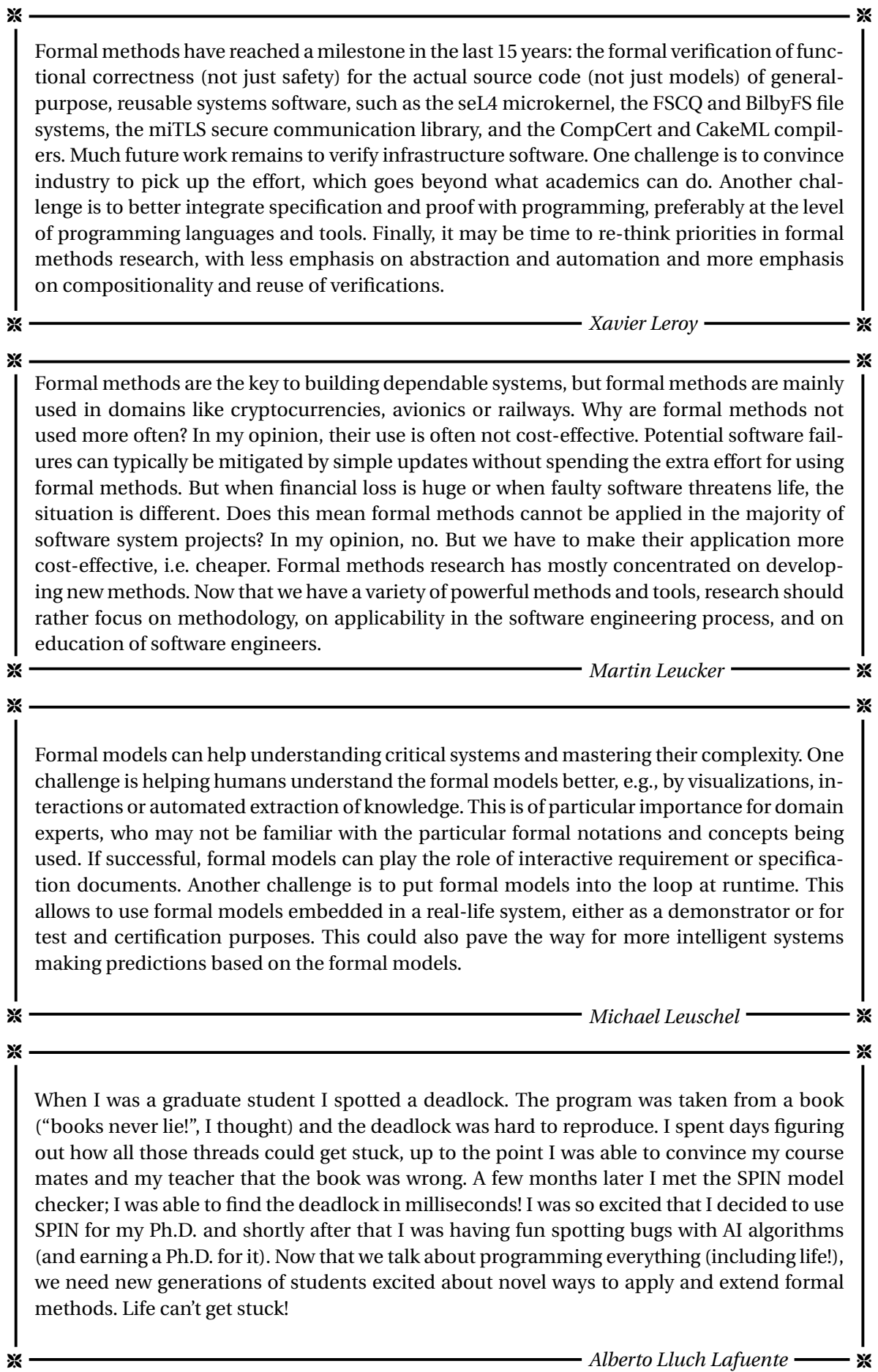




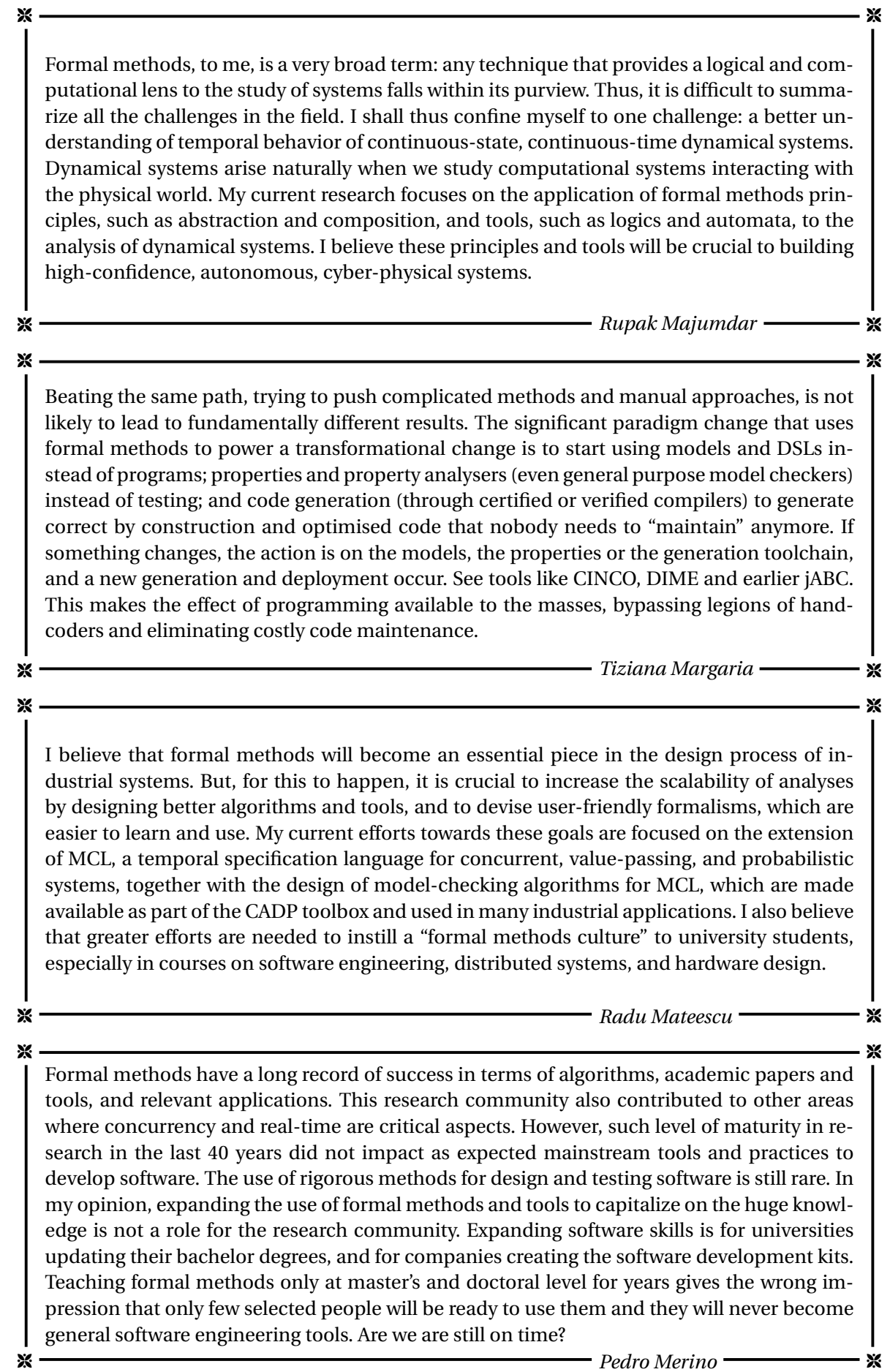




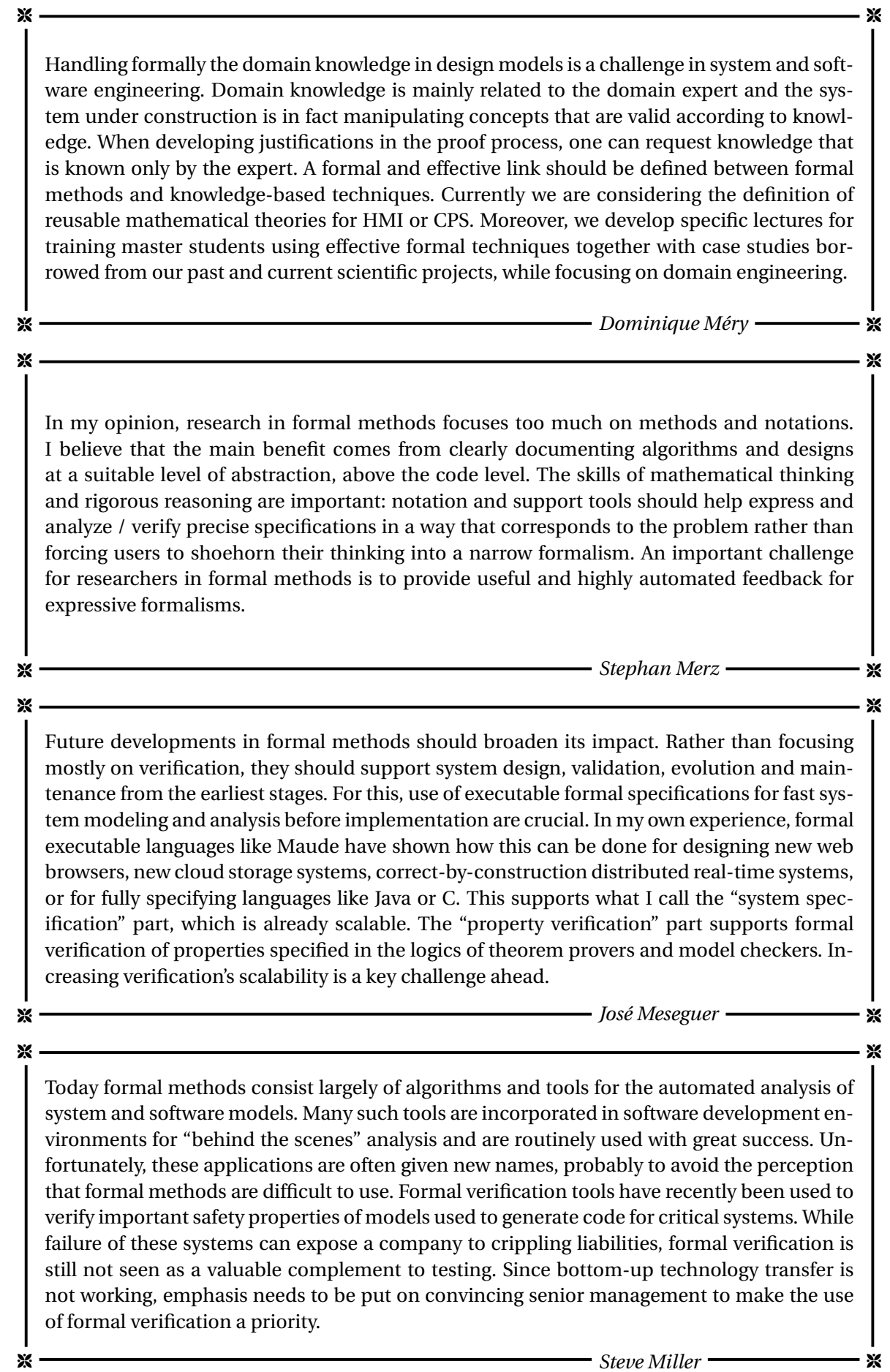




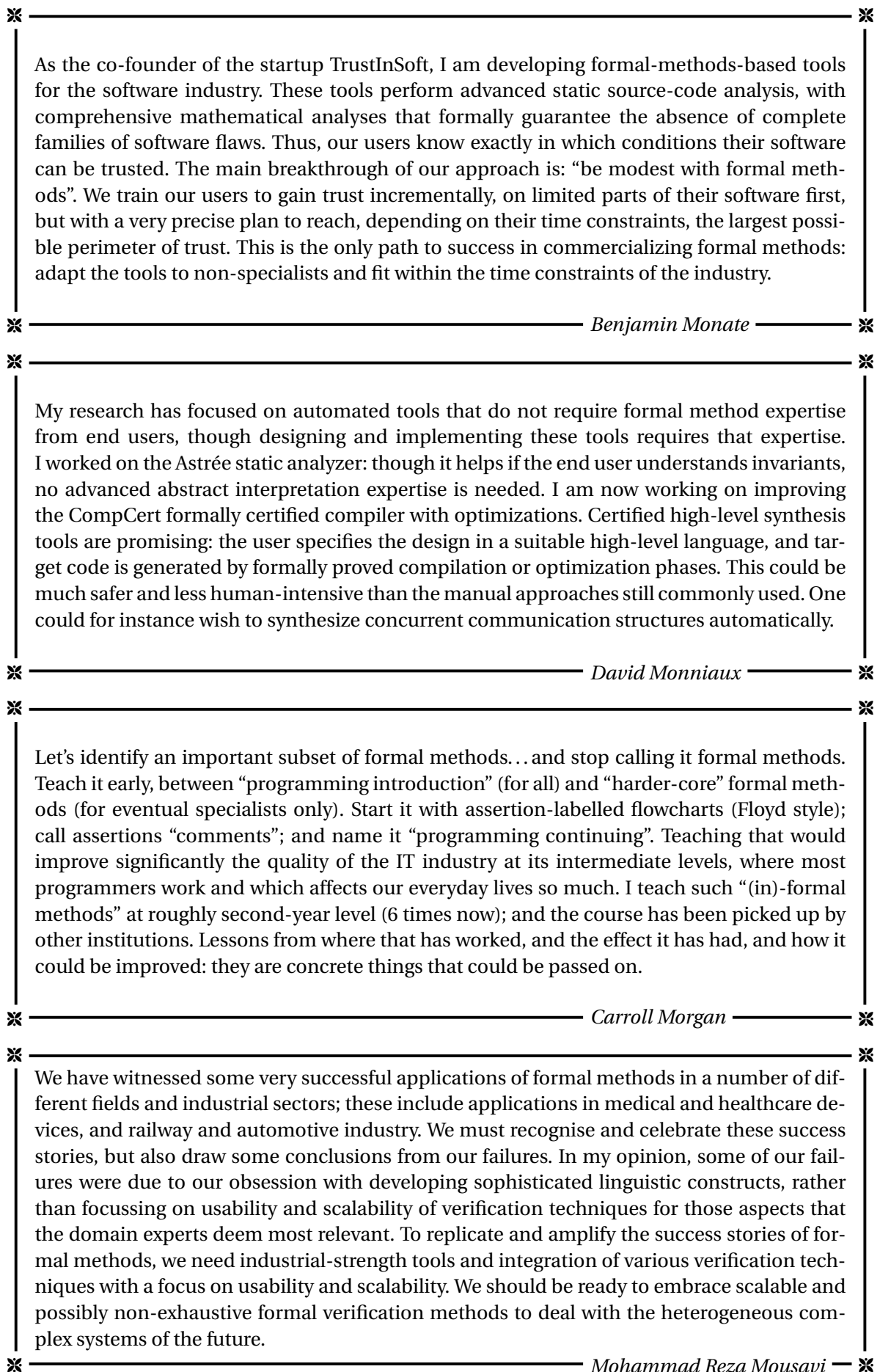




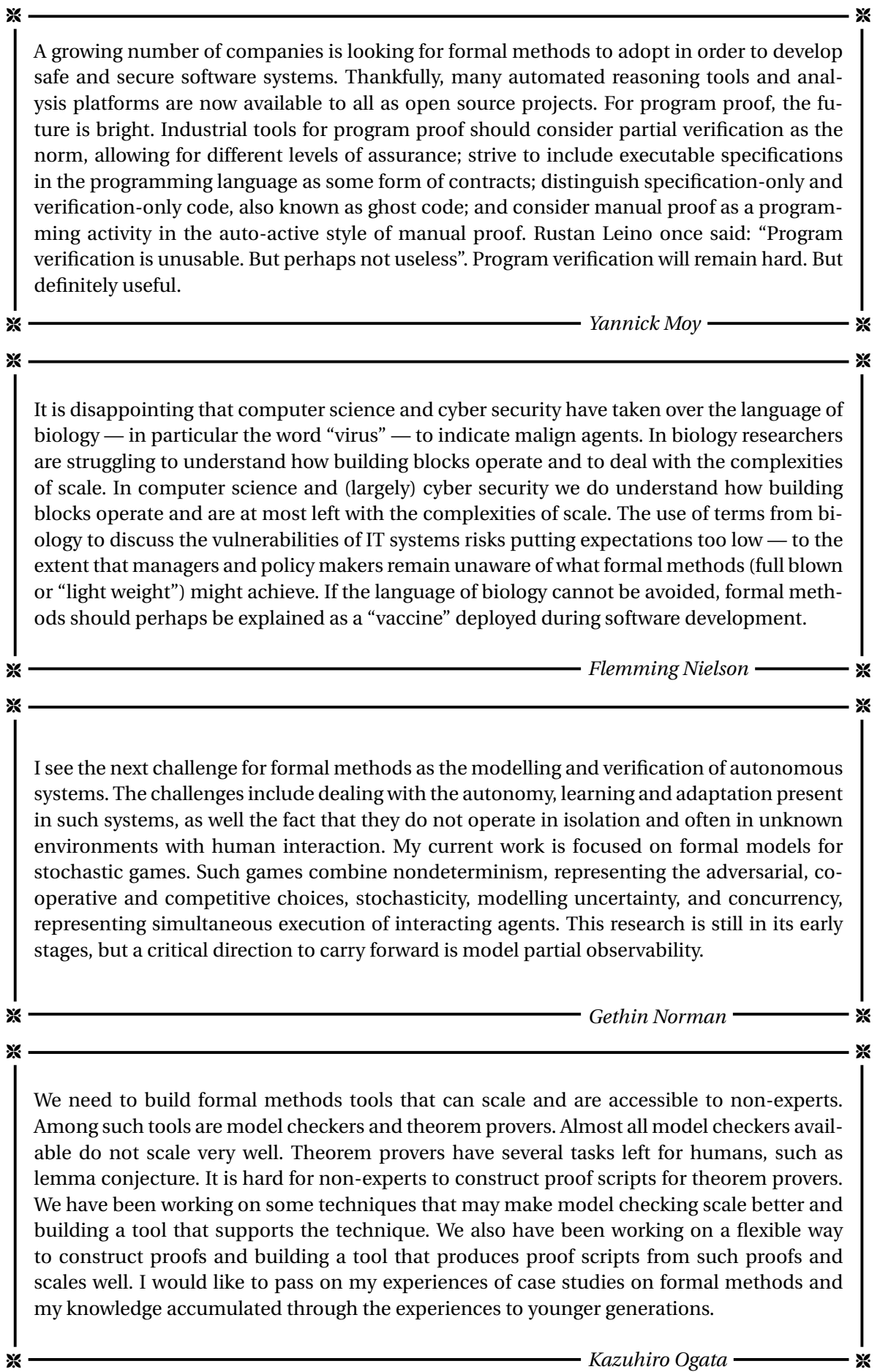




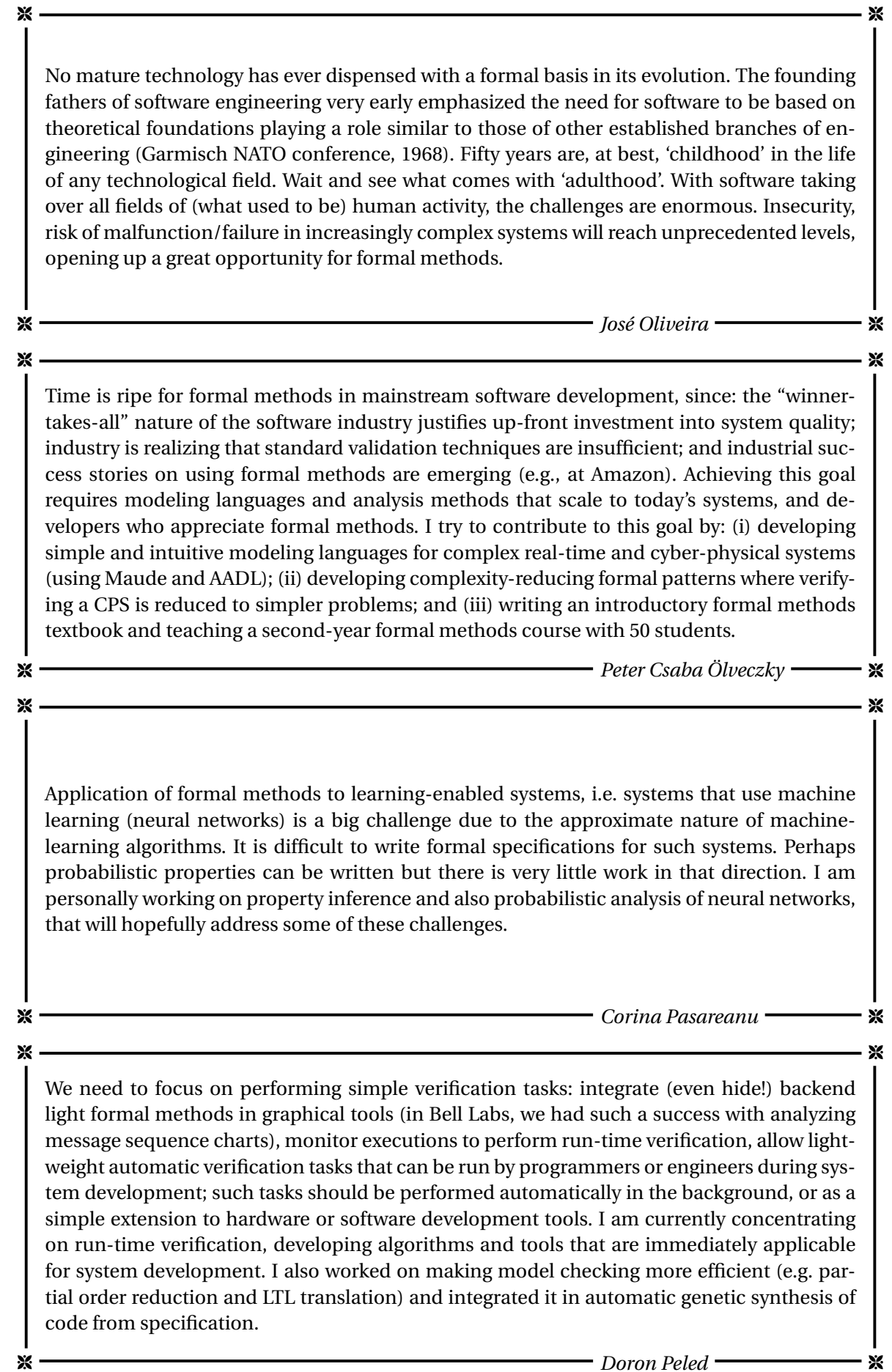




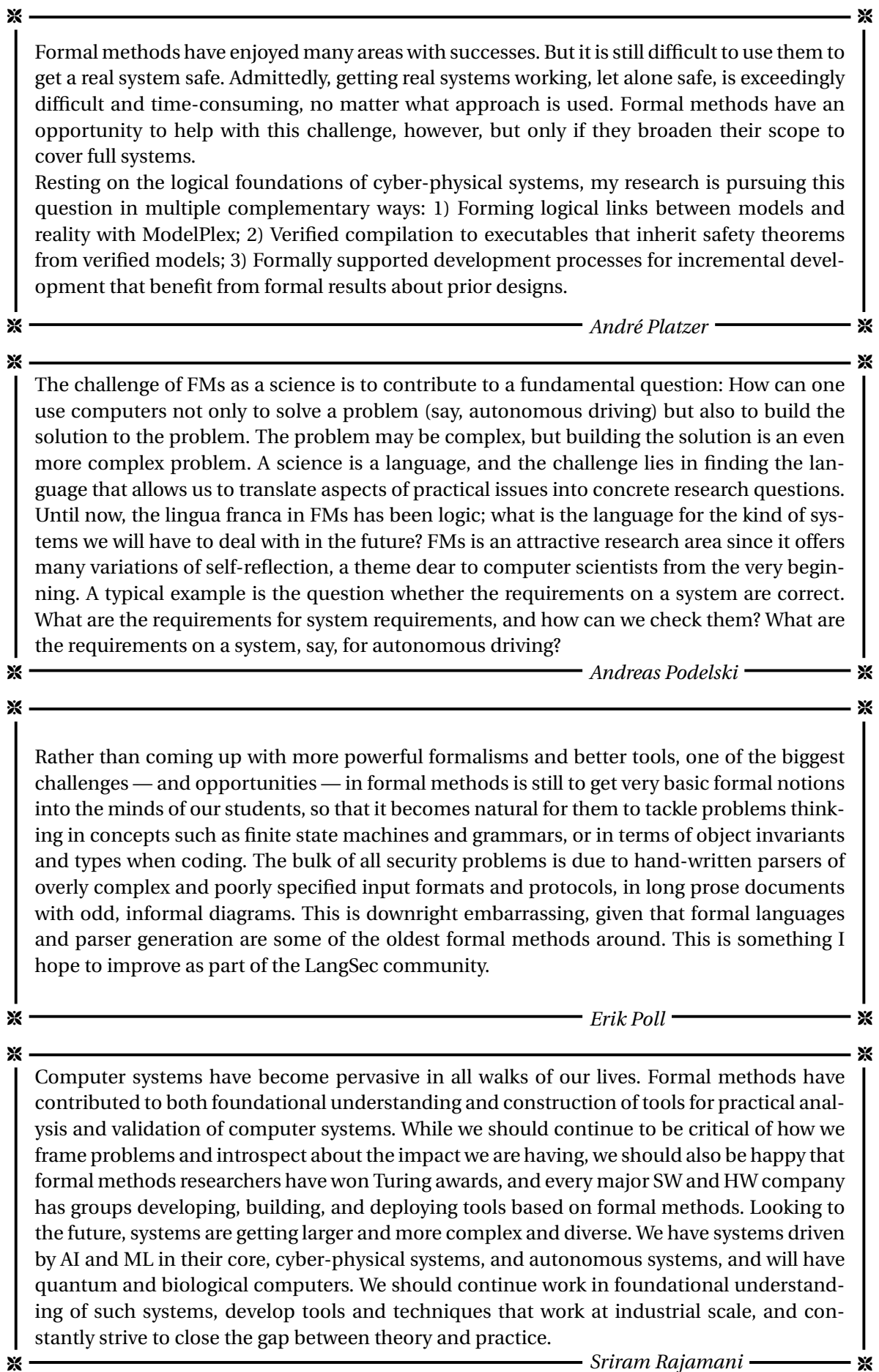




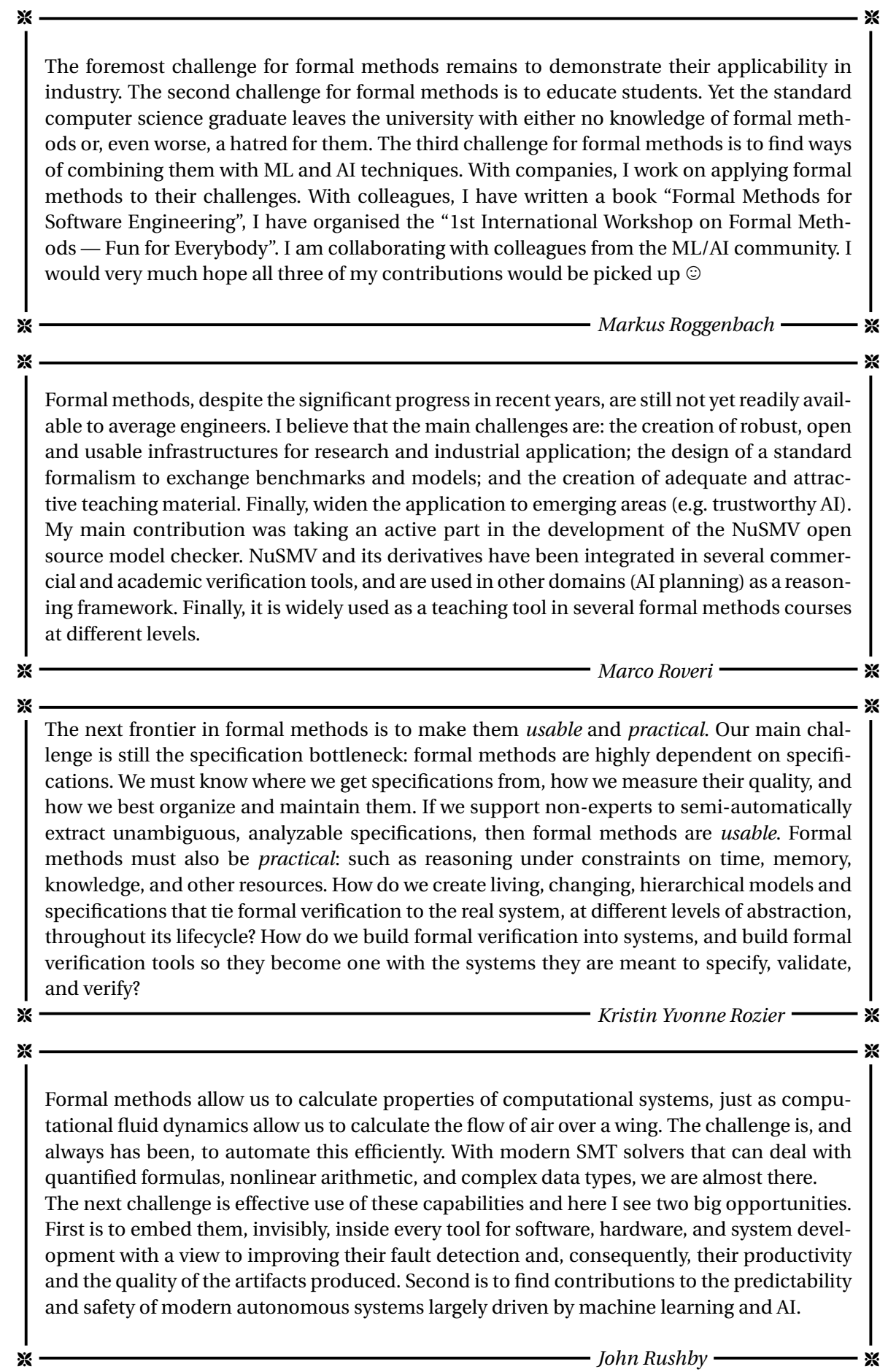




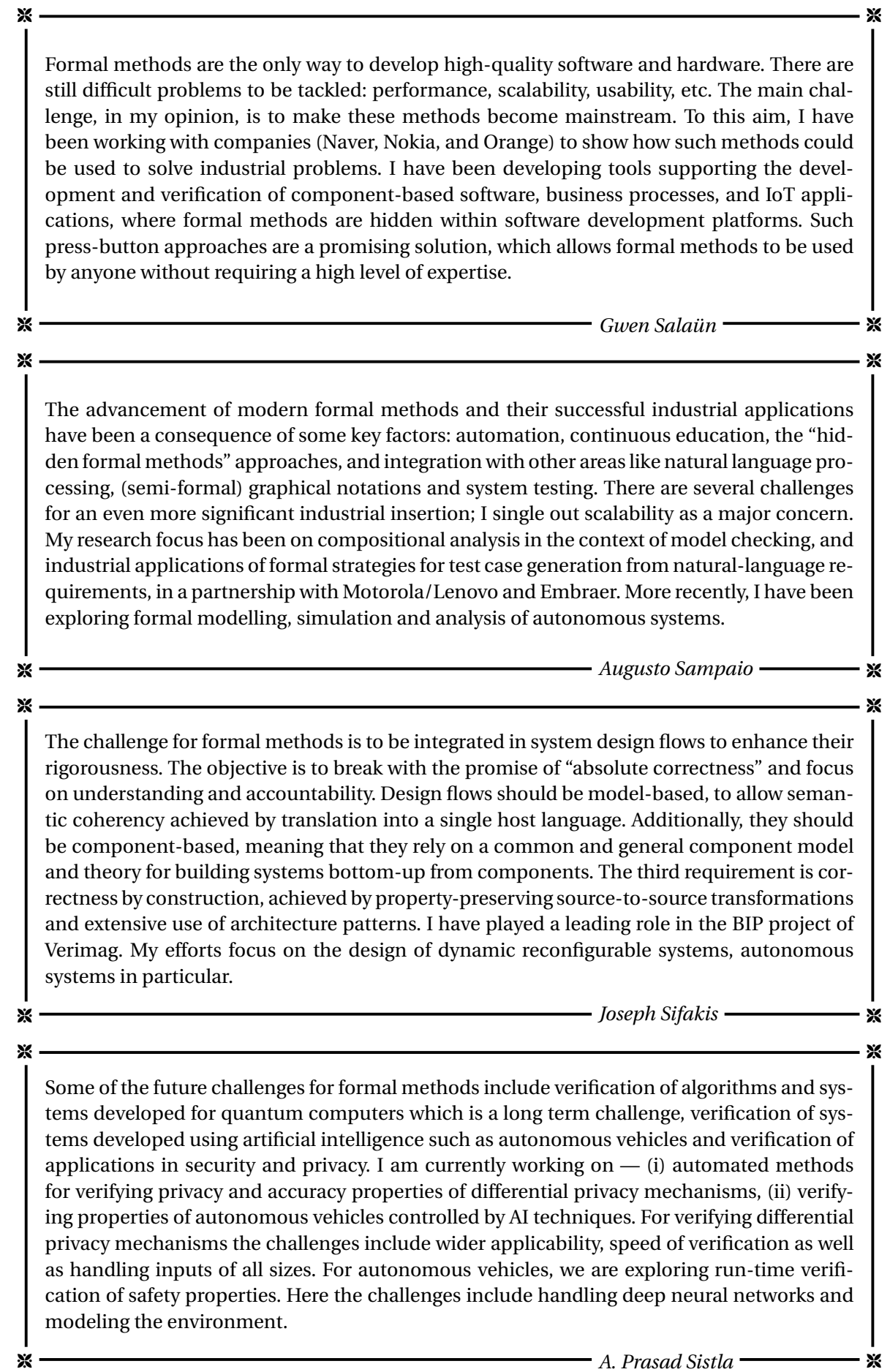




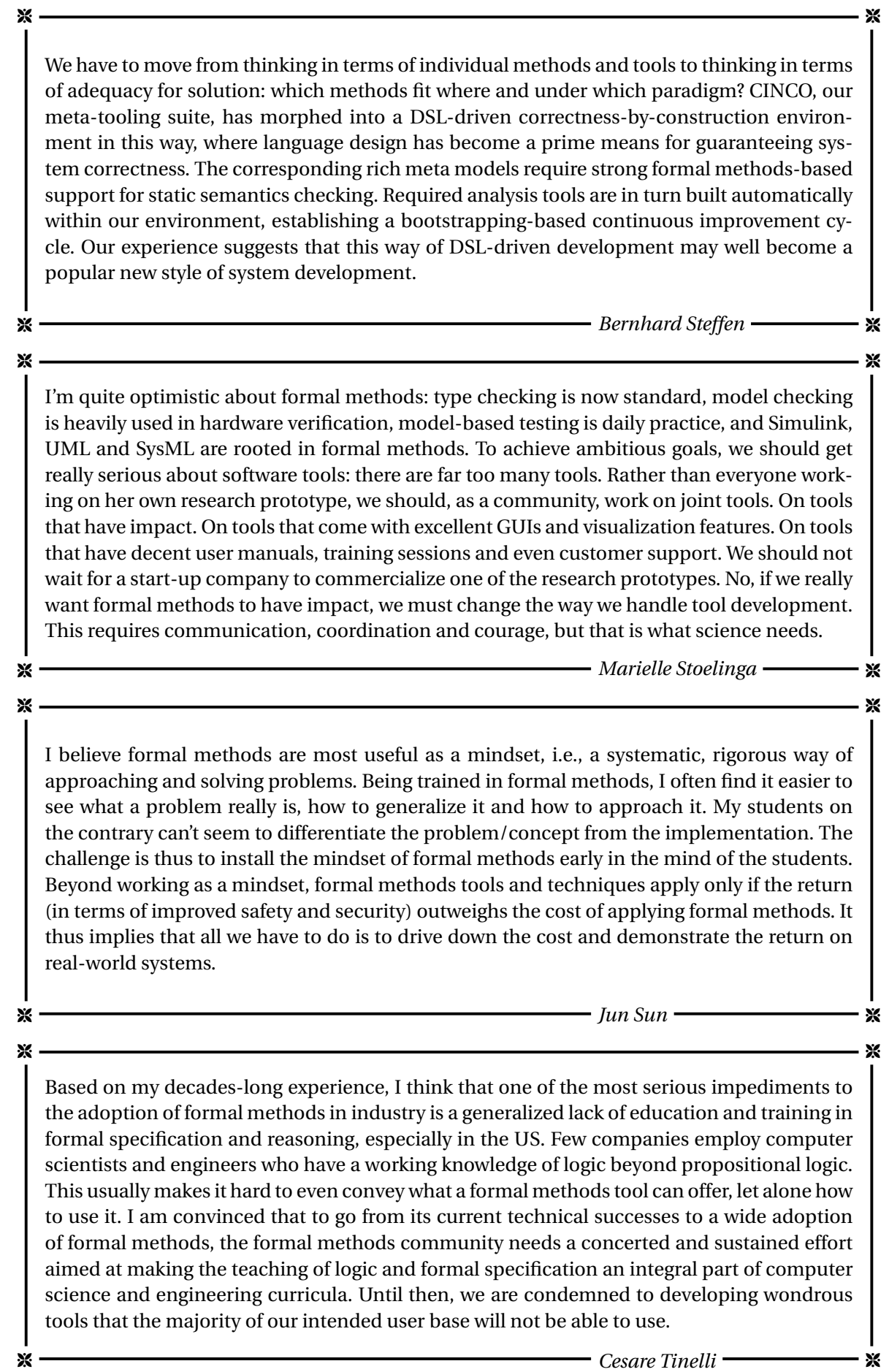




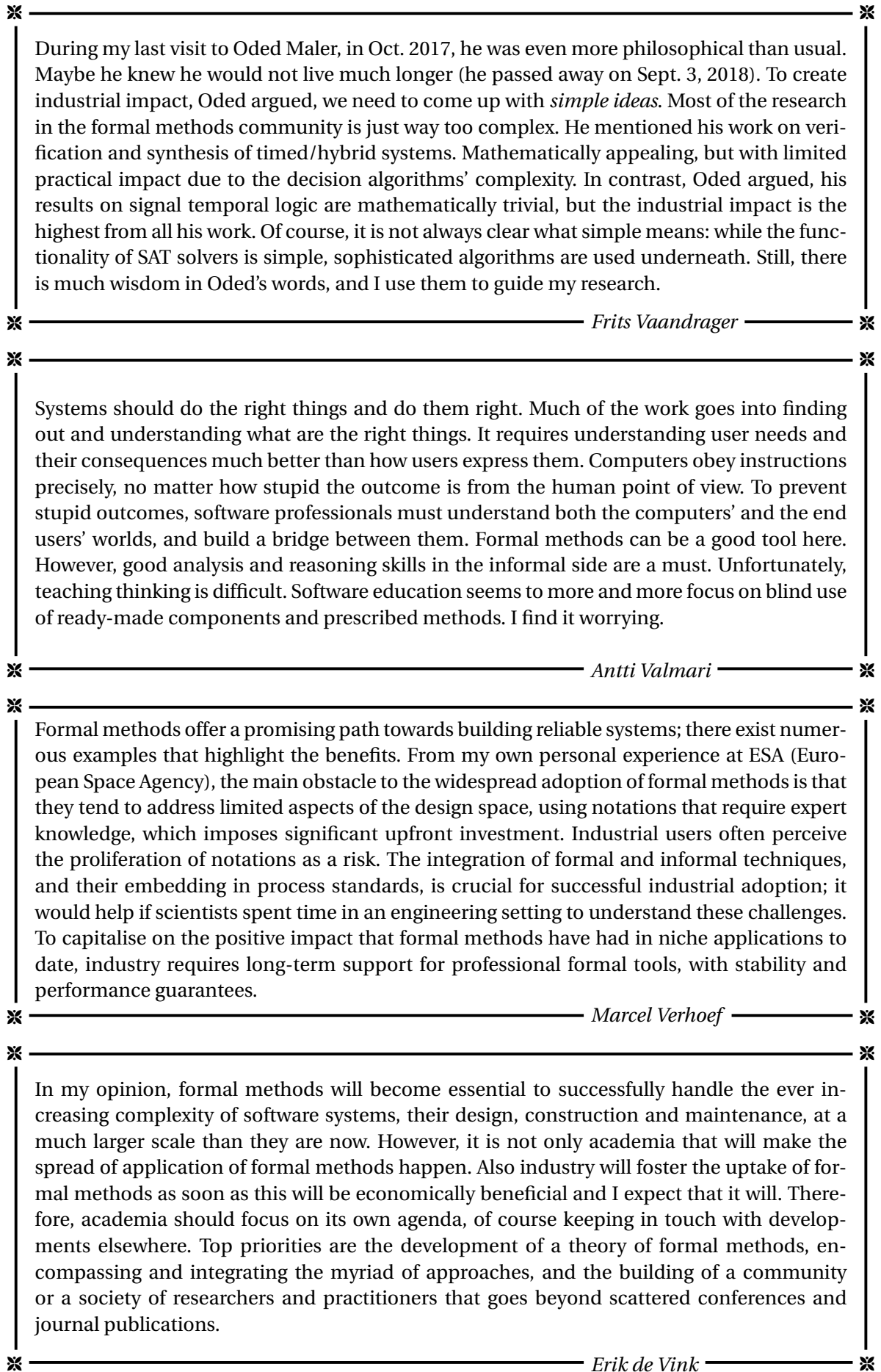




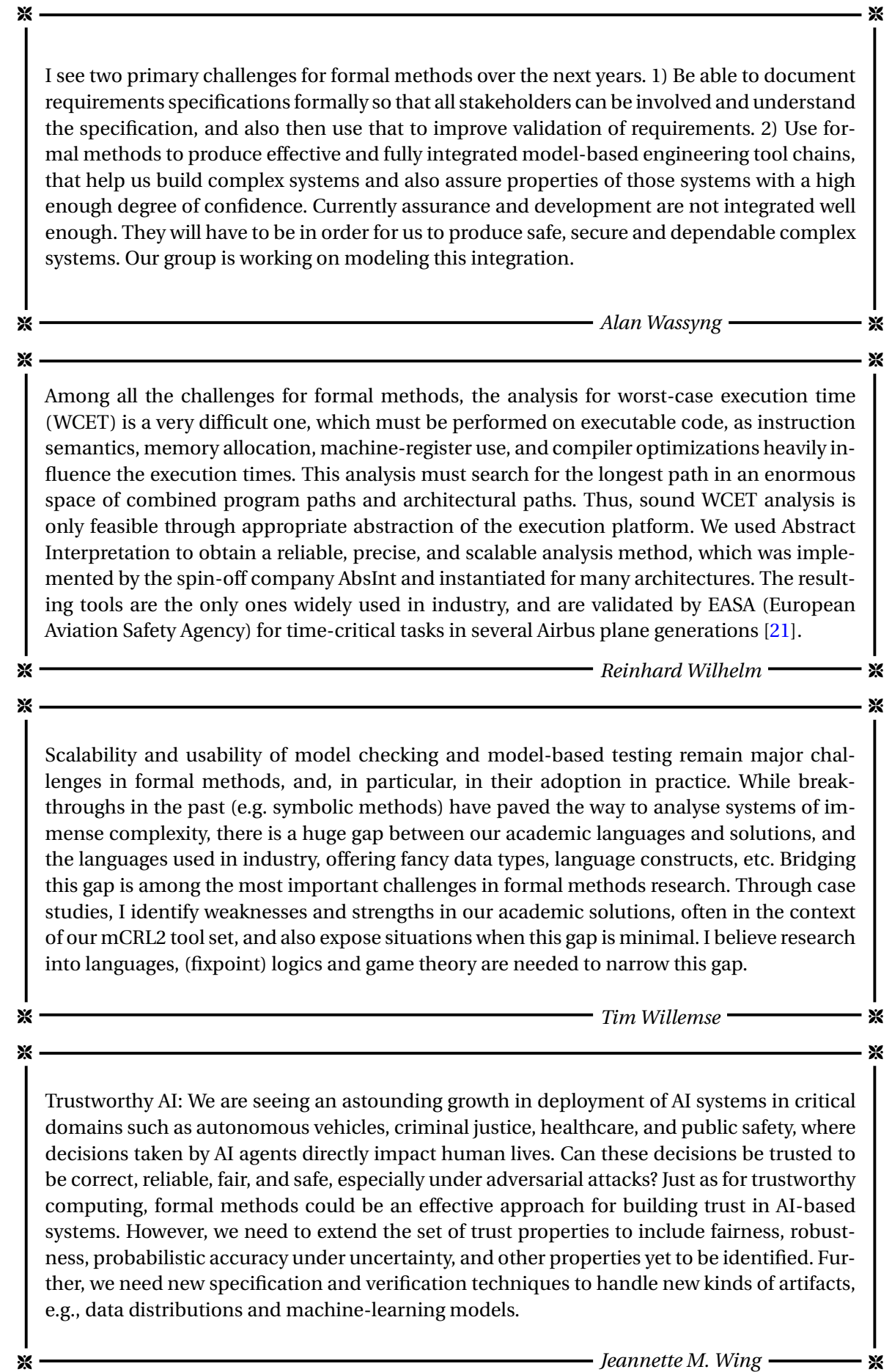




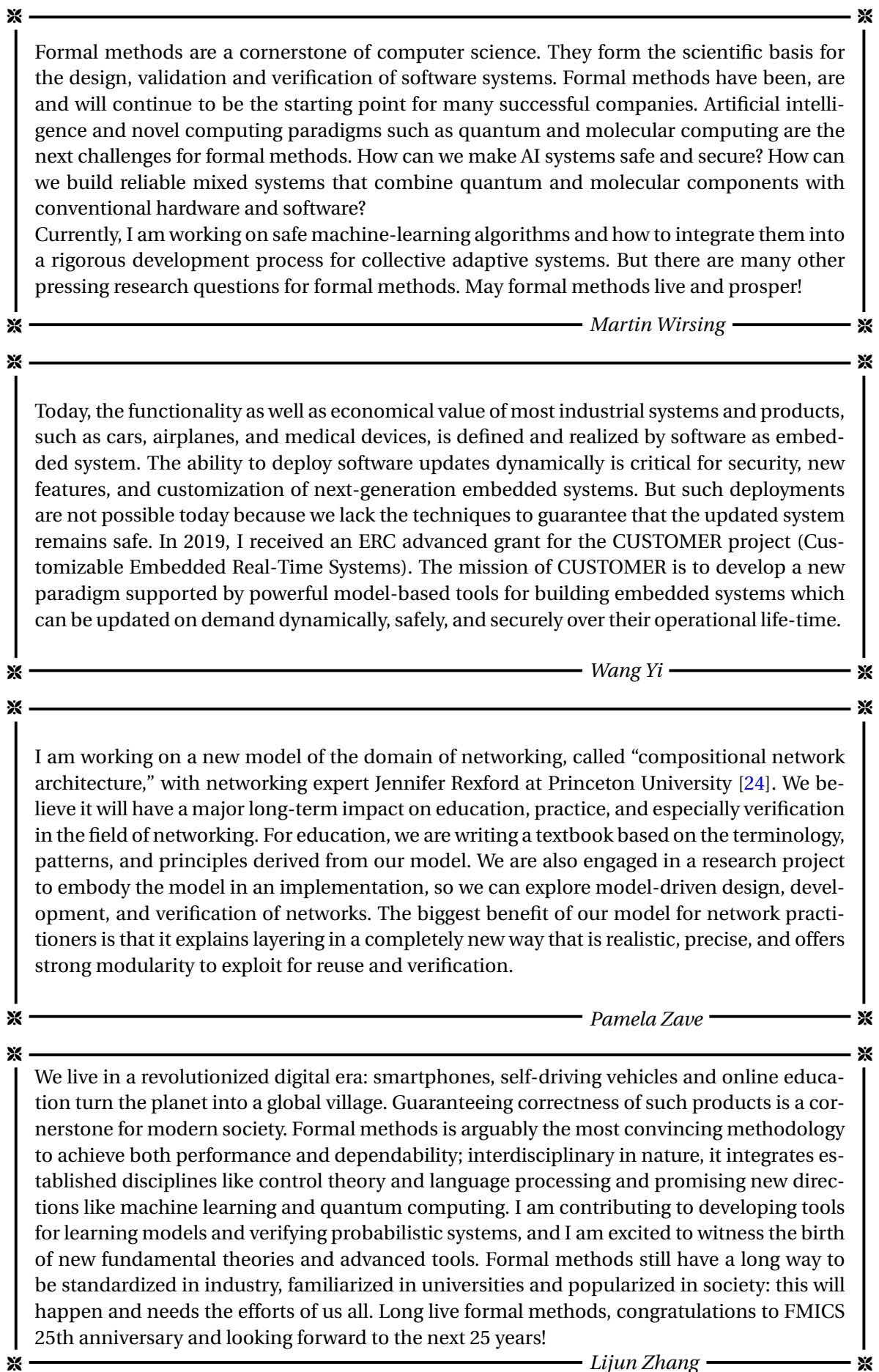




\section{Acknowledgements}

We heartily thank all our colleagues who participated in the survey. This includes all those whose position statement appears in Section 9. Thanks also are due to those experts who took the time to answer our questionnaire, but did not provide a position statement, namely Bernhard Aichernig, Roderick Bloem, Arne Borälv, Rocco De Nicola, Cindy Eisner, Dimitra Giannakopoulou, Georges Gonthier, Susanne Graf, Aarti Gupta, Thomas Henzinger, Holger Hermanns, Michael Hinchey, César Muñoz, Tobias Nipkow, Joël Ouaknine, Charles Pecheur, Alastair Reid, Ina Schieferdecker, and Jim Woodcock. Finally, we are grateful to Nicolas Amat, Pierre Bouvier, Alessio Ferrari, Arnd Hartmanns, Ajay Krishna, Rom Langerak, Lina Marsso, Franco Mazzanti, and Wendelin Serwe, who tested four successive beta-versions of our questionnaire and provided us with many wise observations; Pierre Bouvier, Alessio Ferrari, Dejan Ničković, and Wendelin Serwe also proof-checked the author version of the present report.

\section{References}

1. AbsInt: Astrée software (2020), http://www.absint.com/astree

2. Bartocci, E., Beyer, D., Black, P.E., Fedyukovich, G., Garavel, H., Hartmanns, A., Huisman, M., Kordon, F., Nagele, J., Sighireanu, M., Steffen, B., Suda, M., Sutcliffe, G., Weber, T., Yamada, A.: TOOLympics 2019: An overview of competitions in formal methods. In: Beyer, D., Huisman, M., Kordon, F., Steffen, B. (eds.) Proceedings of the 25th International Conference on Tools and Algorithms for the Construction and Analysis of Systems (TACAS'19), Prague, Czech Republic. Lecture Notes in Computer Science, vol. 11429, pp. 3-24. Springer (Apr 2019). https://doi.org/10.1007/978-3-030-17502-3_1

3. Basile, D., ter Beek, M.H., Fantechi, A., Gnesi, S., Mazzanti, F., Piattino, A., Trentini, D., Ferrari, A.: On the industrial uptake of formal methods in the railway domain: A survey with stakeholders. In: Furia, C.A., Winter, K. (eds.) Proceedings of the 14th International Conference on Integrated Formal Methods (iFM'18), Maynooth, Ireland. Lecture Notes in Computer Science, vol. 11023, pp. 20-29. Springer (Sep 2018). https://doi.org/10.1007/978-3-319-98938-9_2

4. ter Beek, M.H., Borälv, A., Fantechi, A., Ferrari, A., Gnesi, S., Löfving, C., Mazzanti, F.: Adopting formal methods in an industrial setting: The railways case. In: ter Beek, M.H., McIver, A., Oliveira, J.N. (eds.) Proceedings of the 3rd World Congress on Formal Methods (FM'19), Porto, Portugal. Lecture Notes in Computer Science, vol. 11800, pp. 762-772. Springer (Oct 2019). https:// doi.org/10.1007/978-3-030-30942-8_46

5. Bjørner, D., Havelund, K.: 40 years of formal methods - some obstacles and some possibilities? In: Jones, C., Pihlajasaari, P., Sun, J. (eds.) Proceedings of the 19th International Symposium on Formal Methods (FM'14), Singapore. Lecture Notes in Computer Science, vol. 8442, pp. 42-61. Springer (May 2014). https://doi.org/10.1007/978-3-319-06410-9_4

6. Bowen, J.P., Hinchey, M.G.: Ten commandments of formal methods. IEEE Comput. 28(4), 56-63 (Apr 1995). https://doi.org/10.1109/2.375178

7. Cerone, A., Roggenbach, M., Davenport, J., Denner, C., Farrell, M., Haveraaen, M., Moller, F., Körner, P., Krings, S., Ölveczky, P., Schlingloff, B.H., Shilov, N., Zhumagambetov, R.: Rooting formal methods within higher education curricula for computer science and software engineering: A white paper. In: Cerone, A., Roggenbach, M. (eds.) FMFun 2019, Revised Selected Papers. Communications in Computer and Information Science, Springer (2020), to appear 
8. Clarke, E.M., Wing, J.M.: Formal methods: State of the art and future directions. ACM Comput. Surv. 28(4), 626-643 (1996). https:// doi.org/10.1145/242223.242257

9. Davis, J.A., Clark, M.A., Cofer, D.D., Fifarek, A., Hinchman, J., Hoffman, J.A., Hulbert, B.W., Miller, S.P., Wagner, L.G.: Study on the barriers to the industrial adoption of formal methods. In: Pecheur, C., Dierkes, M. (eds.) Proceedings of the 18th International Workshop on Formal Methods for Industrial Critical Systems (FMICS'13), Madrid, Spain. Lecture Notes in Computer Science, vol. 8187, pp. 63-77. Springer (Sep 2013). https://doi.org/10.1007/978-3-642-41010-9_5

10. Ferrari, A., Mazzanti, F., Basile, D., ter Beek, M.H., Fantechi, A.: Comparing formal tools for system design: a judgment study. In: Proceedings of the 42nd International Conference on Software Engineering (ICSE'20). pp. 62-74. ACM (2020). https://doi.org/10.1145/3377811.3380373

11. Garavel, H., Graf, S.: Formal methods for safe and secure computer systems. BSI Study 875, Bundesamt für Sicherheit in der Informationstechnik, Bonn, Germany (Dec 2013), https://www.bsi.bund.de/DE/Publikationen/Studien/Formal_Methods_Study_ 875/study_875.html

12. Garavel, H., Mateescu, R.: Reflections on Bernhard Steffen's physics of software tools. In: Margaria, T., Graf, S., Larsen, K.G. (eds.) Models, Mindsets, Meta: The What, the How, and the Why Not? Lecture Notes in Computer Science, vol. 11200, pp. 186-207. Springer (Jun 2019). https://doi.org/10.1007/978-3-030-22348-9_12

13. Gates, B.: Trustworthy computing (Jan 2002), https://www.wired.com/2002/01/ bill-gates-trustworthy-computing, e-mail memo to Microsoft employees

14. Gnesi, S., Margaria, T. (eds.): Formal methods for industrial critical systems: A survey of applications. Wiley (2013). https://doi.org/10.1002/9781118459898

15. Hall, A.: Seven myths of formal methods. IEEE Softw. 7(5), 11-19 (Sep 1990). https://doi.org/10.1109/52.57887

16. Huisman, M., Gurov, D., Malkis, A.: Formal methods: From academia to industrial practice. A travel guide. CoRR abs/2002.07279 (2020), https://arxiv.org/abs/2002.07279

17. Jones, C.B., Thomas, M.: The development and deployment of formal methods in the UK. CoRR abs/2006.06327 (2020), https://arxiv.org/abs/2006.06327, submitted to Annals of the History of Computing

18. Miller, S.P.: Lessons from twenty years of industrial formal methods. In: Proceedings of the 20th High Confidence Software and Systems Conference (HCSS’12) (2012), http://cps-vo. org/node/3434

19. Rushby, J.: Formal methods and the certification of critical systems. Tech. Rep. SRI-CSL93-7, Computer Science Laboratory, SRI International, Menlo Park, CA (Dec 1993), http: //www.csl.sri.com/papers/csl-93-7/, also issued under the title "Formal Methods and Digital Systems Validation for Airborne Systems" as NASA Contractor Report 4551, Dec 1993

20. Steffen, B.: The physics of software tools: SWOT analysis and vision. International Journal on Software Tools for Technology Transfer 19(1), 1-7 (2017). https://doi.org/10.1007/s10009-016-0446-x

21. Wilhelm, R.: Real time spent on real time. Commun. ACM (Oct 2020), to appear

22. Wing, J.M.: A specifier's introduction to formal methods. IEEE Computer 23(9), 8-22 (Sep 1990). https://doi.org/10.1109/2.58215

23. Woodcock, J., Larsen, P.G., Bicarregui, J., Fitzgerald, J.: Formal methods: Practice and experience. ACM Computing Surveys 41(4), 19:1-19:36 (2009). https://doi.org/10.1145/1592434.1592436

24. Zave, P., Rexford, J.: The compositional architecture of the Internet. Commun. ACM 62(3), 78-87 (Feb 2019). https:// doi.org/10.1145/3226588 